\title{
The formation and evolution of hydrogen-deficient post-AGB white dwarfs: The emerging chemical profile and the expectations for the PG 1159-DB-DQ evolutionary connection
}

\author{
L. G. Althaus ${ }^{1, \star}$, A. M. Serenelli ${ }^{2}$, J. A. Panei ${ }^{3,4, \star \star}$, A. H. Córsico ${ }^{3,4, \star \star \star}$, E. García-Berro ${ }^{1,5}$, and C. G. Scóccola ${ }^{3, \dagger}$ \\ ${ }^{1}$ Departament de Física Aplicada, Universitat Politècnica de Catalunya, Av. del Canal Olímpic s/n, 08860 Castelldefels, \\ Barcelona, Spain \\ e-mail: althaus@fcaglp.unlp.edu.ar \\ 2 Institute for Advanced Study, School of Natural Sciences, Einstein Drive, Princeton, NJ 08540, USA \\ e-mail: aldos@ias.edu \\ 3 Facultad de Ciencias Astronómicas y Geofísicas, Universidad Nacional de La Plata, Paseo del Bosque S/N, (B1900FWA) \\ La Plata, Argentina \\ e-mail: [panei; acorsico; cscoccola]@fcaglp.unlp.edu.ar \\ ${ }^{4}$ Instituto de Astrofísica La Plata, IALP, CONICET-UNLP \\ 5 Institut d'Estudis Espacials de Catalunya, Ed. Nexus, c/Gran Capità 2, 08034 Barcelona, Spain \\ e-mail: garcia@fa.upc.es
}

Received 6 September 2004 / Accepted 30 January 2005

\begin{abstract}
We explore the formation and evolution of hydrogen-deficient post-AGB white dwarfs. To this end, we compute the complete evolution of an initially $2.7 M_{\odot}$ star from the zero-age main sequence through the thermally pulsing and mass-loss phases to the white dwarf stage. Particular attention is given to the chemical abundance changes during the whole evolution. A time-dependent scheme for the simultaneous treatment of abundance changes caused by nuclear reactions, diffusive overshooting, salt fingers and convection is considered. We employed the double-diffusive mixing-length theory of convection for fluids with composition gradients. The study can therefore be considered as a test of its performance in low-mass stars. Also, timedependent element diffusion for multicomponent gases is taken into account during the white dwarf evolution. The evolutionary stages corresponding to the last helium thermal pulse on the early white-dwarf cooling branch and the following born-again episode are carefully explored. Relevant aspects for PG 1159 stars and DB white dwarf evolution are studied in the framework of these new evolutionary models that take into account the history of the white dwarf progenitor. The scope of the calculations is extended to the domain of the helium-rich, carbon-contaminated DQ white dwarfs with the aim of exploring the plausibility of the evolutionary connection PG 1159-DB-DQ. In this regard, the implications for the double-layered chemical structure in pulsating DB white dwarfs is investigated. We examine the consequences of mass-loss episodes during the PG 1159 stage for the chemical stratification of the outer layer of DB and DQ white dwarfs.
\end{abstract}

Key words. stars: evolution - stars: abundances - stars: AGB stars: interiors - stars: white dwarfs - stars: oscillations

\section{Introduction}

White-dwarf stars with helium-rich atmospheres, commonly referred to as DB white dwarfs, comprise about $20 \%$ of the total white dwarf population. Most of these stars are widely believed to be the result of a born-again episode, which is considered to be the most promising scenario to explain the existence of hydrogen-deficient post-asymptotic-giant-branch (post-AGB)

$\star$ Member of the Carrera del Investigador Científico y Tecnológico and IALP, CONICET / FCAG-UNLP, Argentina.

$\star \star$ Fellow of CONICET, Argentina.

$\star \star \star$ Member of the Carrera del Investigador Científico y Tecnológico, CONICET, Argentina.

$\dagger$ Fellow of CONICET, Argentina. stars - see, for instance, Fujimoto (1977), Schönberner (1979) and Iben et al. (1983) for earlier references. Other possible channels that could lead to the formation of DB white dwarfs could involve the class of helium-rich, supergiant $\mathrm{R}$ Coronae Borealis ( $\mathrm{RCrB})$ stars, probably linked to the hot hydrogendeficient $\mathrm{O}(\mathrm{He})$ stars, and the hydrogen-poor, carbon-normal star evolution directly from the extended horizontal branch (AGB manqué stars such as SdB stars) into the white dwarf state (Schönberner 1996; Werner 2001). Within the born-again scenario, a very late helium-shell flash is experienced by a white-dwarf remnant during its early cooling phase after hydrogen burning has almost ceased. At the beginning of this thermal pulse, most of the residual hydrogen envelope is engulfed 
by the helium-flash convection zone and completely burnt. The star is then forced to evolve rapidly back to the AGB and finally into the central star of a planetary nebula at high effective temperatures $\left(T_{\text {eff }}\right)$ but now as a hydrogen-deficient, quiescent helium-burning object. Such objects are expected to exhibit surface layers that are substantially enriched with the products of helium burning, particularly carbon.

Important observed examples of these hydrogen-deficient post-AGB stars are the very hot PG 1159 and their probable progenitors, the Wolf-Rayet type central stars of planetary nebulae having spectral type $[\mathrm{WC}]^{1}$ (Koesterke \& Hamann 1997; Dreizler \& Heber 1998; Werner 2001). Indeed, spectroscopic analyses have revealed that most of these post-AGB stars are characterized by hydrogen-deficient and helium-, carbon- and oxygen-rich surface abundances. In particular, the appreciable abundance of oxygen in the atmospheres of these stars has been successfully explained by Herwig et al. (1999) on the basis of evolutionary calculations of the born-again scenario that incorporate convective overshoot.

Strong observational evidence suggests that PG 1159 stars are the direct predecessors of the majority of helium-rich DO stars which are the hot and immediate progenitors of DB white dwarfs (Dreizler \& Werner 1996; Dreizler \& Heber 1998). Theoretical evidence for the existence of an evolutionary link between PG 1159 stars and most of the DO white dwarfs has been presented by Unglaub \& Bues (2000) on the basis of diffusion calculations with mass loss for hot white dwarfs. In addition, evolutionary calculations taking into account time-dependent element diffusion (Dehner \& Kawaler 1995; Gautschy \& Althaus 2002) have shown that, as a result of gravitational settling of carbon and oxygen, the PG 1159-like initial chemical stratification of a pre-white dwarf evolves into a superficially helium dominated double-layered chemical structure when the domain of the pulsating DBs is reached. In fact, two different chemical transition zones would characterize the envelope of the PG 1159 descendants: a still uniform intershell region which is rich in helium, carbon and oxygen; the relics of the short-lived mixing episode that occurred during the last helium thermal pulse, and an overlying pure helium mantle which thickens as cooling proceeds. These works clearly foster the plausibility of an evolutionary connection between most of the PG 1159 and DB stars.

The shape of the outer layer chemical profile is a matter of the utmost importance as far as the pulsational properties of DB white dwarfs are concerned. In fact, the presence of a diffusioninduced double-layered chemical structure has been shown by Fontaine \& Brassard (2002) to have strong implications for the theoretical pulsational spectrum of these stars. According to these authors, asteroseismological inference about the core composition of DBs and the ${ }^{12} \mathrm{C}(\alpha, \gamma){ }^{16} \mathrm{O}$ reaction rate based on single-layered DB models (with a pure helium envelope atop a carbon-oxygen core) should be taken with a pinch of salt. More recently, DB asteroseismological fittings incorporating both the double-layered envelope feature expected from time-dependent diffusion calculations and adjustable carbon-oxygen cores have been presented by Metcalfe et al. (2003) for a wide range of

\footnotetext{
${ }^{1}$ Not to be confused with massive Wolf-Rayet stars.
}

helium contents and stellar masses. Despite their models yielding significantly better fits to the observations, the derived stellar parameters for some fits lead them to conclude that doublelayered models with adjustable carbon-oxygen cores may not be entirely appropriate to explain the observations.

The possibility that PG 1159 stars could eventually evolve into DB white dwarfs that are characterized by envelopes with a single composition transition zone has recently been explored by Althaus \& Córsico (2004). Indeed, on the basis of evolutionary calculations that incorporate time-dependent element diffusion, Althaus \& Córsico (2004) have shown that if the helium content in PG 1159 stars is smaller than $\approx 10^{-3} M_{*}$, the double-layered structure is expected to become single-layered by the time evolution has proceeded to the domain of the variable DBs. Although the small quoted value for the helium content is difficult to reconcile with evolutionary calculations for the formation of hydrogen-deficient post-AGB, which predict the total helium mass left in the star to be about $M_{\mathrm{He}} \approx 10^{-2} M_{*}$ (Herwig et al. 1999), the existence of PG 1159 stars with low helium content cannot be discarded. In fact, mass-loss rates ranging from $10^{-7}$ to $10^{-8} M_{\odot} / \mathrm{yr}$ are observed in many luminous PG 1159 stars. In addition, tentative evidence for the persistence of mass-loss rates within the range $10^{-7}$ to $10^{-10} M_{\odot} / \mathrm{yr}$ down to the domain of hot helium-rich white dwarfs has been presented (Werner 2001). The existence of such mass-loss rates would imply that most of the helium-rich envelope of DB progenitors could be substantially reduced during the time interval mass loss would be operative. It is worth mentioning that the existence of PG 1159 stars with a helium content as low as $10^{-3} M_{\odot}$ has been suggested by asteroseismology in at least one of these stars with a stellar mass of $0.6 M_{\odot}$ (Kawaler \& Bradley 1994), thus implying the occurrence of modest massloss during the evolution to the PG 1159 phase.

In this work we explore some aspects relevant for the evolution of DB white dwarfs on the basis of new evolutionary calculations that account for a complete and self-consistent treatment of the evolutionary stages prior to white dwarf formation. We concentrate on DB white dwarfs resulting from a born-again episode. Specifically, we follow the evolution of an initially $2.7 M_{\odot}$ star from the zero-age main sequence through the thermally pulsing and mass-loss phases on the AGB to the white dwarf regime. The evolutionary stages corresponding to the almost complete burning of protons following the occurrence of the very late thermal pulse and the ensuing born-again episode are carefully explored. Attention is paid to the abundance changes during the entire evolution, which are described by means of a time-dependent scheme for the simultaneous treatment of nuclear evolution and mixing processes due to convection, salt fingers and diffusive overshoot. We emphasize in particular the role of time-dependent element diffusion in the chemical abundance distribution in the white-dwarf regime. We also investigate the influence of mass-loss episodes during the PG 1159 and DO phases for the chemical stratification of pulsating DBs. Finally, we extend the scope of our calculations to the domain of the helium-rich carbon-contaminated DQ white dwarfs, the supposed cooler descendants of DBs. The plausibility of the evolutionary connection PG 1159-DB-DQ 
(Fontaine \& Brassard 2002) is assessed in the framework of our new evolutionary models.

As far as we are aware, this is the first time that the evolution of hydrogen-deficient white dwarfs is modelled consistently on the basis of a complete and detailed treatment of the physical processes that lead to the formation of such stars. At this point it is important to note that we think that a reexamination of the pulsational properties of variable DB white dwarfs deserves to be performed in the frame of the new evolutionary models presented in this work. However, being this issue important it is also true that this would carry us too far afield. The paper is organized as follows. The following section contains the main physical inputs to the models, particularly regarding the treatment of the chemical abundance changes. In Sect. 3 we present the evolutionary results. There, we elaborate on the main aspects of pre-white dwarf evolution, particularly during the born-again phase and the attendant chemical changes. We also describe the results corresponding to the PG 1159 and white-dwarf regimes. In Sect. 4, we discuss the implications of our results for the white dwarf evolution as well as the role played by mass-loss episodes. Section 5 is devoted to discuss some concluding remarks.

\section{Input physics and evolutionary sequences}

The calculations presented in this work have been done using a Henyey- type stellar evolution code. In particular, we employed the LPCODE evolutionary code that is specifically designed to compute the formation and evolution of white dwarf stars, following a star from the main sequence through the thermal pulses and post-AGB phases. Except for minor modifications, the code is essentially that described at length in Althaus et al. (2003) and references therein. LPCODE uses OPAL radiative opacities (including carbon- and oxygen-rich compositions) for different metallicities (Iglesias \& Rogers 1996), complemented, at low temperatures, with the molecular opacities from Alexander \& Ferguson (1994). Opacities for different metallicities are required in particular during the white dwarf regime, where metallicity gradients induced by gravitational settling develop in the envelope of such stars. High-density conductive opacities are those of Itoh et al. (1994) and the references cited there, whereas neutrino emission rates are those of Itoh (1997) and references therein. The equation of state for the low-density regime includes partial ionization for hydrogen and helium compositions, radiation pressure and ionic contributions. For the high-density regime, partially degenerate electrons and Coulomb interactions are also considered. During the born-again episode, the remnant star develops surface layers rich in helium, carbon and oxygen. In that case, we employ an ideal equation of state that includes partial ionization for any mixture of the three chemical species. Finally, for the white dwarf regime, we considered an updated version of the equation of state of Magni \& Mazzitelli (1979).

The nuclear network employed in LPCODE accounts explicitly for the following 16 elements: ${ }^{1} \mathrm{H},{ }^{2} \mathrm{H},{ }^{3} \mathrm{He},{ }^{4} \mathrm{He}$, ${ }^{7} \mathrm{Li},{ }^{7} \mathrm{Be},{ }^{12} \mathrm{C},{ }^{13} \mathrm{C},{ }^{14} \mathrm{~N},{ }^{15} \mathrm{~N},{ }^{16} \mathrm{O},{ }^{17} \mathrm{O},{ }^{18} \mathrm{O},{ }^{19} \mathrm{~F},{ }^{20} \mathrm{Ne}$ and ${ }^{22} \mathrm{Ne}$. In addition, we consider 34 thermonuclear reaction rates to describe the hydrogen (proton-proton chain and $\mathrm{CNO}$ bi-cycle) and helium burning and carbon ignition. Specifically, for hydrogen burning we consider:

$$
\begin{aligned}
& \mathrm{p}+\mathrm{p} \rightarrow{ }^{2} \mathrm{H}+\mathrm{e}^{+}+v \\
& \mathrm{p}+\mathrm{p}+\mathrm{e}^{-} \rightarrow{ }^{2} \mathrm{H}+v \\
& { }^{2} \mathrm{H}+\mathrm{p} \rightarrow{ }^{3} \mathrm{He}+\gamma \\
& { }^{3} \mathrm{He}+{ }^{3} \mathrm{He} \rightarrow \alpha+2 \mathrm{p} \\
& { }^{3} \mathrm{He}+\alpha \rightarrow{ }^{7} \mathrm{Be}+\gamma \\
& { }^{3} \mathrm{He}+\mathrm{p} \rightarrow{ }^{4} \mathrm{He}+\gamma \\
& { }^{7} \mathrm{Be}+\mathrm{e}^{-} \rightarrow{ }^{7} \mathrm{Li}+v \\
& { }^{7} \mathrm{Li}+\mathrm{p} \rightarrow 2 \alpha \\
& { }^{7} \mathrm{Be}+\mathrm{p} \rightarrow 2 \alpha \\
& { }^{12} \mathrm{C}+\mathrm{p} \rightarrow{ }^{13} \mathrm{C}+\mathrm{e}^{+}+v \\
& { }^{13} \mathrm{C}+\mathrm{p} \rightarrow{ }^{14} \mathrm{~N}+\gamma \\
& { }^{14} \mathrm{~N}+\mathrm{p} \rightarrow{ }^{15} \mathrm{~N}+\mathrm{e}^{+}+v \\
& { }^{15} \mathrm{~N}+\mathrm{p} \rightarrow{ }^{12} \mathrm{C}+\alpha \\
& { }^{15} \mathrm{~N}+\mathrm{p} \rightarrow{ }^{16} \mathrm{O}+\gamma \\
& { }^{16} \mathrm{O}+\mathrm{p} \rightarrow{ }^{17} \mathrm{O}+\mathrm{e}^{+}+v \\
& { }^{17} \mathrm{O}+\mathrm{p} \rightarrow{ }^{18} \mathrm{O}+\mathrm{e}^{+}+v \\
& { }^{17} \mathrm{O}+\mathrm{p} \rightarrow{ }^{14} \mathrm{~N}+\alpha \\
& { }^{18} \mathrm{O}+\mathrm{p} \rightarrow{ }^{15} \mathrm{~N}+\alpha \\
& { }^{18} \mathrm{O}+\mathrm{p} \rightarrow{ }^{19} \mathrm{~F}+\gamma \\
& { }^{19} \mathrm{~F}+\mathrm{p} \rightarrow{ }^{16} \mathrm{O}+\alpha \\
& { }^{19} \mathrm{~F}+\mathrm{p} \rightarrow{ }^{20} \mathrm{Ne}+\gamma .
\end{aligned}
$$

For helium burning, the reaction rates taken into account are:

$$
\begin{aligned}
& 3 \alpha \rightarrow{ }^{12} \mathrm{C}+\gamma \\
& { }^{12} \mathrm{C}+\alpha \rightarrow{ }^{16} \mathrm{O}+\gamma \\
& { }^{13} \mathrm{C}+\alpha \rightarrow{ }^{16} \mathrm{O}+n \\
& { }^{14} \mathrm{~N}+\alpha \rightarrow{ }^{18} \mathrm{O}+\mathrm{e}^{+}+\gamma \\
& { }^{15} \mathrm{~N}+\alpha \rightarrow{ }^{19} \mathrm{~F}+\gamma \\
& { }^{16} \mathrm{O}+\alpha \rightarrow{ }^{20} \mathrm{Ne}+\gamma \\
& { }^{17} \mathrm{O}+\alpha \rightarrow{ }^{20} \mathrm{Ne}+n \\
& { }^{18} \mathrm{O}+\alpha \rightarrow{ }^{22} \mathrm{Ne}+\gamma \\
& { }^{20} \mathrm{Ne}+\alpha \rightarrow{ }^{24} \mathrm{Mg}+\gamma \\
& { }^{22} \mathrm{Ne}+\alpha \rightarrow{ }^{25} \mathrm{Mg}+n \\
& { }^{22} \mathrm{Ne}+\alpha \rightarrow{ }^{26} \mathrm{Mg}+\gamma .
\end{aligned}
$$

This set of nuclear reactions allows us to follow in detail the main nucleosynthesis occurring during the thermally pulsing AGB and born-again phases. The reactions for carbon burning are:

$$
\begin{aligned}
& { }^{12} \mathrm{C}+{ }^{12} \mathrm{C} \rightarrow{ }^{20} \mathrm{Ne}+\alpha \\
& { }^{12} \mathrm{C}+{ }^{12} \mathrm{C} \rightarrow{ }^{24} \mathrm{Mg}+\gamma .
\end{aligned}
$$

Nuclear reaction rates are taken from Caughlan \& Fowler (1988), except for the reactions ${ }^{15} \mathrm{~N}(p, \gamma)^{16} \mathrm{O}$, $\left.\left.{ }^{15} \mathrm{~N}(p, \alpha)\right)^{12} \mathrm{C}, \quad{ }^{18} \mathrm{O}(p, \alpha)\right)^{15} \mathrm{~N}, \quad{ }^{18} \mathrm{O}(p, \gamma){ }^{19} \mathrm{~F}, \quad{ }^{12} \mathrm{C}(\alpha, \gamma){ }^{16} \mathrm{O}$, ${ }^{16} \mathrm{O}(\alpha, \gamma){ }^{20} \mathrm{Ne},{ }^{13} \mathrm{C}(\alpha, n){ }^{16} \mathrm{O},{ }^{18} \mathrm{O}(\alpha, \gamma){ }^{22} \mathrm{Ne},{ }^{22} \mathrm{Ne}(\alpha, n){ }^{25} \mathrm{Mg}$ and ${ }^{22} \mathrm{Ne}(\alpha, \gamma)^{26} \mathrm{Mg}$, which are taken from Angulo et al. (1999). The $\left.{ }^{12} \mathrm{C}(\alpha, \gamma)\right)^{16} \mathrm{O}$ reaction rate given by Angulo et al. (1999) is about twice as large as that of Caughlan \& Fowler (1988). 
An important aspect of the present study is the treatment of the abundance changes throughout the different evolutionary phases. In particular, during some short-lived phases of the evolution, for instance during the born-again episode and also the thermally pulsing AGB phase, the nuclear time-scale of some reactions becomes comparable to the convective mixing time-scale. In this case the instantaneous mixing approximation turns out to be completely inadequate for addressing chemical mixing in convective regions. A more physically sound chemical evolution scheme than instantaneous mixing is therefore required. In this work, we have considered a time-dependent scheme for the simultaneous treatment of chemical changes caused by nuclear burning and mixing processes. Specifically, abundance changes are described by the set of equations

$$
\left(\frac{\mathrm{d} \boldsymbol{Y}}{\mathrm{d} t}\right)=\left(\frac{\partial \boldsymbol{Y}}{\partial t}\right)_{\mathrm{nuc}}+\frac{\partial}{\partial M_{r}}\left[\left(4 \pi r^{2} \rho\right)^{2} D \frac{\partial \boldsymbol{Y}}{\partial M_{r}}\right]
$$

with $\boldsymbol{Y}$ being the vector containing the number fraction of all considered elements. Details about the numerical procedure for solving this equation can be found in Althaus et al. (2003). Here, mixing due to convection, salt fingers and overshoot is treated as a diffusion process (second term of Eq. (1)). The efficiency of convective and salt-finger mixing is described by appropriate diffusion coefficients $D$ which are specified by our treatment of convection. In particular, we considered the extended mixing-length theory (MLT) of convection for fluids with composition gradients developed by Grossman et al. (1993) in its local approximation as given by Grossman \& Taam (1996). These authors have developed the non-linear MLT of double-diffusive convection ${ }^{2}$ that applies in convective, semiconvective and salt-finger instability regimes. In this work, the mixing length free parameter $\alpha$ is taken to be 1.5 . To our knowledge, our work constitutes the first application of the extended MLT to full evolutionary calculation of low-mass stars. The first term of Eq. (1) gives the abundance changes due to thermonuclear reactions. This term is linearized following the implicit scheme developed by Arnett \& Truran (1969). In our treatment of abundance changes, nuclear evolution is fully coupled to the current composition change due to the various mixing processes - see Althaus et al. (2003) for additional details. In LPCODE, abundance changes are performed for the 16 elements described above after the convergence of each stellar model (and not during iterations). For a better integration precision in the nuclear evolution, each evolutionary time step is divided into 5 chemical time steps.

Mixing episodes beyond what is predicted by the Schwarzschild criterion for convective stability strongly affect the inner chemical profile of white dwarf progenitors. The occurrence of such mixing episodes, particularly core overshooting and/or semiconvection, is suggested by both theoretical and observational evidence. Recently, Straniero et al. (2003) have presented an assessment of the inner chemical abundances in a $3 M_{\odot}$ model star resulting from different mixing processes occurring during the late stage of core helium burning phase. In particular, they conclude that models which incorporate

\footnotetext{
2 The effects of both thermal and composition gradients determine the stability of the fluid.
}

semiconvection or a moderate overshoot (related to the inertia of the moving fluid elements) applied to core and convective shells predict a sharp variation of the chemical composition in the carbon-oxygen core. On the other hand, the presence of overshooting below the convective envelope during the thermal pulses has been shown by Herwig et al. (1997) to yield third dredge-up and carbon-rich AGB stars for relatively low initial mass progenitors - see also Ventura et al. (1999) and Mazzitelli et al. (1999). In addition, overshooting below the helium-flash convection zone during the thermally pulsing AGB phase gives rise to intershell abundances in agreement with abundance determinations in hydrogen-deficient post-AGB remnants such as PG 1159 stars (Herwig et al. 1999; Herwig 2000). In view of these considerations, we have allowed for some overshoot in our work. In particular, we have included time-dependent overshoot mixing during all evolutionary stages. Our scheme for the changes in the abundances allows for a self-consistent treatment of diffusive overshooting in the presence of nuclear burning. We have considered exponentially decaying diffusive overshooting above and below any formally convective region, including the convective core (main sequence and central helium burning phases), the external convective envelope and the short-lived helium-flash convection zone which develops during the thermal pulses and born-again episode. In particular, the expression for the diffusion coefficient in overshoot regions is $D_{\mathrm{OV}}=D_{\mathrm{C}} \exp \left(-2 z / H_{\mathrm{v}}\right)$ where $D_{\mathrm{C}}$ is the diffusion coefficient at the edge of the convection zone, $z$ is the radial distance from the boundary of the convection zone, $H_{\mathrm{v}}=f H_{\mathrm{P}}$, where the free parameter $f$ is a measure of the extent of the overshoot region, and $H_{\mathrm{P}}$ is the pressure scale height at the convective boundary. In this study we have adopted $f=0.015$, which accounts for the observed width of the main sequence and abundances in hydrogen-deficient, post-AGB objects (Herwig et al. 1997, 1999; Herwig 2000; Mazzitelli et al. 1999). The breathing pulse instability occurring towards the end of core helium burning has been suppressed - see Straniero et al. (2003) for a recent discussion of this point.

The evolution of the chemical abundance distribution caused by diffusion processes during the white dwarf regime has also been taken into account in this work. Our timedependent element diffusion treatment, based on the formulation for multicomponent gases presented by Burgers (1969), considers gravitational settling, chemical and thermal diffusion but not radiative levitation, which has been neglected, for the nuclear species ${ }^{1} \mathrm{H},{ }^{3} \mathrm{He},{ }^{4} \mathrm{He},{ }^{12} \mathrm{C},{ }^{13} \mathrm{C},{ }^{14} \mathrm{~N},{ }^{16} \mathrm{O}$ and ${ }^{22} \mathrm{Ne}$ (Althaus et al. 2003). Diffusion velocities are evaluated at each evolutionary step. During the white dwarf regime, the metal mass fraction $Z$ in the envelope is not assumed to be fixed, instead it is specified consistently according to the prediction of element diffusion.

Given the considerable load of computing time demanded by our self-consistent solution of nuclear evolution and timedependent mixing, we have limited ourselves to examining only one case for the evolution for the progenitor star. Specifically, we compute the evolution of an initially $2.7 M_{\odot}$ stellar model from the zero-age main sequence all the way from the stages of hydrogen and helium burning in the core up to the tip of the AGB where helium thermal pulses occur. 


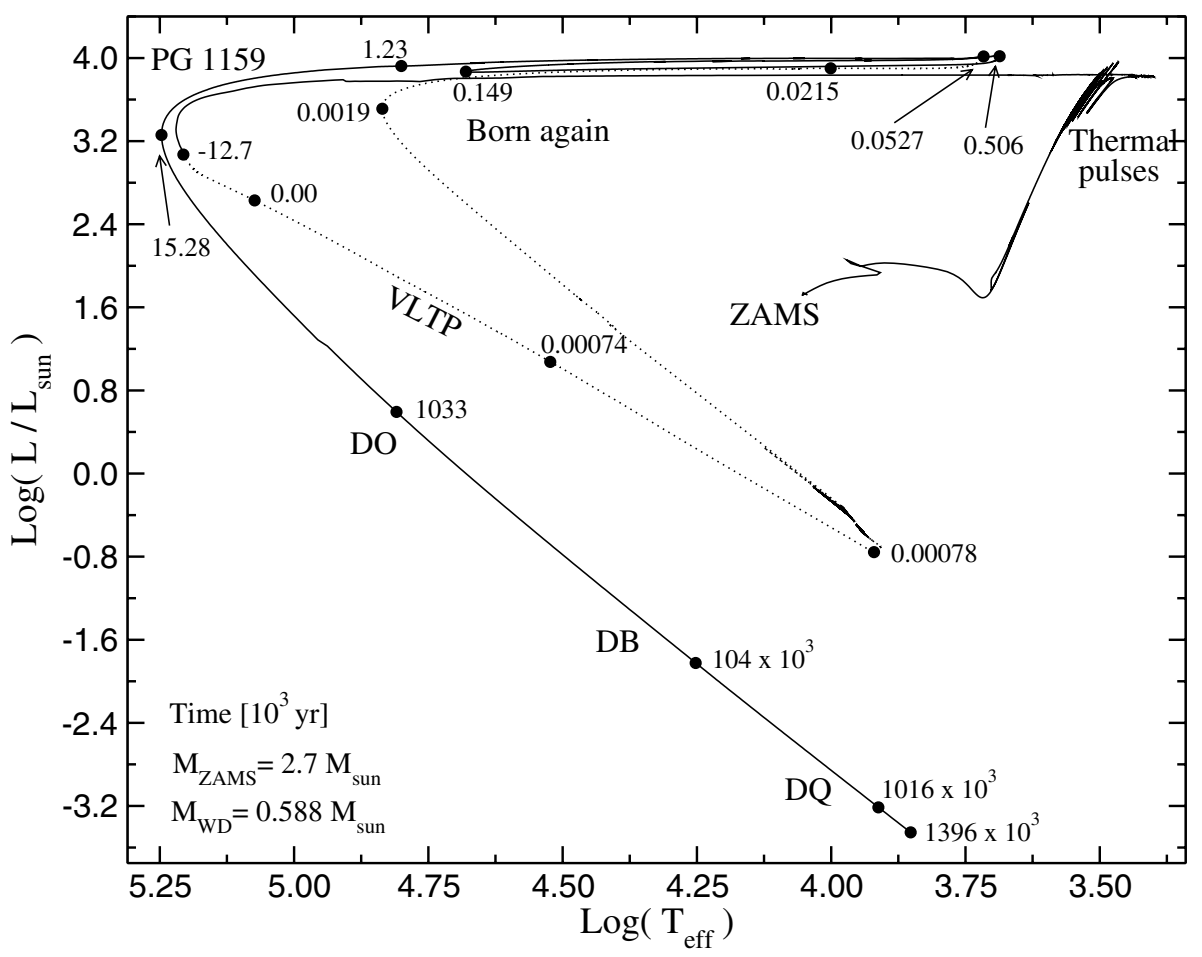

Fig. 1. Hertzsprung-Russell diagram for the complete evolution of our $2.7 M_{\odot}$ stellar model from the ZAMS to the white dwarf domain. The star experiences a very last thermal pulse (VLTP) on its early cooling phase after hydrogen burning has almost ceased. The following evolutionary stages correspond to the born-again phase and are depicted, together with the VLTP stage with dotted lines. The numbers close to the filled dots along the evolutionary track correspond to the age (in $10^{3} \mathrm{yr}$ ) counted from the occurrence of the peak of the last thermal pulse. The domain of the PG 1159, DO, DB and DQ stars are indicated, as well as the thermal pulse and born-again stages. As a result of mass loss episodes, the stellar mass decreases from 2.7 to $0.5885 M_{\odot}$. After the born-again episode, the hydrogen-deficient post-AGB remnant experiences a second excursion towards lower temperatures before reaching eventually its terminal white dwarf track.

A solar-like initial composition $(Y, Z)=(0.275,0.02)$ has been adopted (Anders \& Grevesse 1989). We have considered massloss episodes taking place during the stages of core helium burning and red giant branch following the usual Reimers formulation with $\eta_{\mathrm{R}}=1$. During the thermally pulsing phase we adopt the mass-loss rates from Blöcker (1995). After experiencing 10 thermal pulses, the progenitor departs from the AGB and evolves towards high effective temperatures. Departure from the AGB has been forced to occur at such an advanced phase of the helium shell flash cycle that the post-AGB remnant undergoes a final thermal pulse during the early white dwarf cooling phase - a very late thermal pulse, see Blöcker (2001) for a review - where most of the residual hydrogen envelope is burnt. As mentioned in the introduction, there exists observational evidence that suggests that some hydrogen-deficient post-AGB stars could experience strong stellar winds, which could reduce the helium content in the star considerably. To assess the influence of such mass-loss episodes for the further evolution we have considered extreme mass-loss rates of $10^{-7}$ and $10^{-8} M_{\odot} /$ yr during the PG 1159 stage.

The evolutionary calculations have been followed down to the domain of the helium-rich carbon-contaminated DQ white dwarfs with the aim of exploring the evolutionary connection between DQ white dwarfs and the PG 1159 stars. The evolutionary sequence from the ZAMS to the white dwarf stage comprises $\approx 70000$ stellar models. Stellar models are divided into about 1500 mesh points. The final mass of the white dwarf remnant is $0.5885 M_{\odot}$. Our models would also be particularly appropriate for pulsational studies of PG 1159 and variable DB white dwarfs. We report below the main results of our calculations.

\section{Evolutionary results}

\subsection{White dwarf progenitor: Evolution from the ZAMS to the thermally pulsing phase}

In Fig. 1 we show the complete Hertzsprung-Russell (HR) diagram. Our numerical simulation covers all the evolutionary phases of an initially $2.7 M_{\odot}$ star from the ZAMS to the domain of the DQ white dwarfs, including the stages corresponding to the helium thermal pulses on the AGB and the bornagain episode (shown as a dotted line). The age (in units of $10^{3} \mathrm{yr}$ ) counted from the occurrence of the last thermal pulse peak on the cooling track is shown at selected points along the evolutionary track. The total time spent in central hydrogen and helium burning is $6.57 \times 10^{8} \mathrm{yr}$. After helium is exhausted in the core and during the following $1.16 \times 10^{7} \mathrm{yr}$, the star evolves towards the thermally pulsing phase on the AGB. There, helium shell burning becomes unstable and the star experiences the well-known recurrent thermal instability 


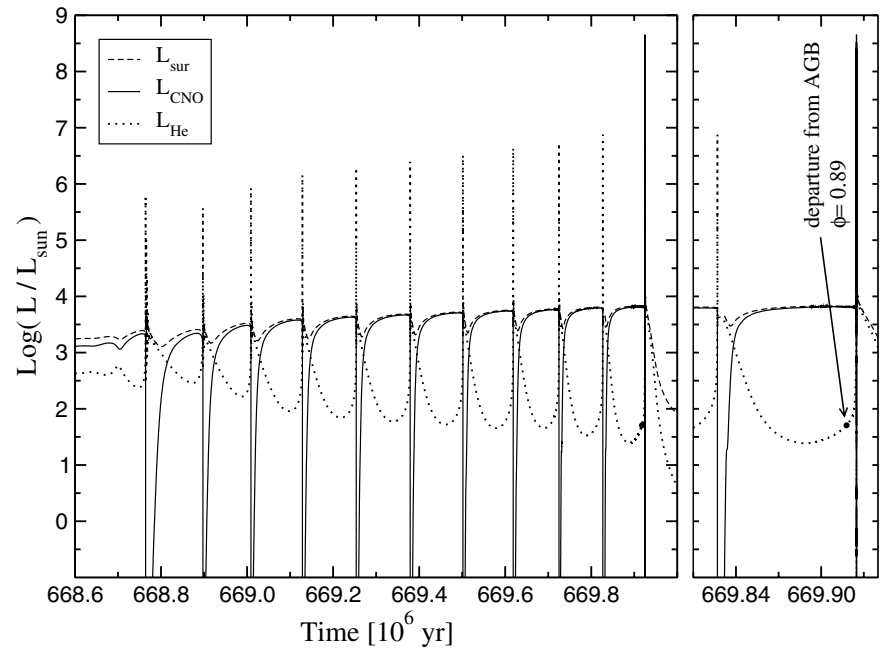

Fig. 2. The temporal evolution of surface luminosity and hydrogenand helium-burning luminosities in solar units for an initially $2.7 M_{\odot}$ star during the thermally pulsing phase on the AGB. After experiencing 10 thermal pulses, the progenitor star departs from the AGB with a thermal-pulse cycle phase of 0.89 (denoted by a black dot). The last thermal pulse shown here takes place already on the white dwarf cooling track, where the hydrogen-burning luminosity exceeds $10^{8} L_{\odot}$. The right panel shows the luminosity evolution after the occurrence of the tenth pulse. Age is given in $10^{6} \mathrm{yr}$ counted from the main sequence.

commonly referred to as helium thermal pulses (Schwarzschild \& Härm 1965). After 10 thermal pulses and as a result of strong mass loss, the remnant star leaves the AGB and evolves towards high $T_{\text {eff }}$ s. This takes place when the luminosity of the star is supported mostly by stationary hydrogen burning. Mass loss decreases the stellar mass from 2.7 to $0.5885 M_{\odot}$. Note that the post-AGB remnant undergoes a further (last) thermal pulse on its early white dwarf cooling track shortly after hydrogen burning has virtually ceased (Blöcker 2001). During this born-again episode, most of the residual hydrogen envelope is engulfed by the deep helium-flash convection zone and is completely burnt. Evolution proceeds through these stages very fast, since it takes only about $30 \mathrm{yr}$ for the remnant to expand from a white dwarf configuration to giant dimensions. Note also that after the born-again episode, the now hydrogen-deficient post-AGB remnant experiences a second excursion towards lower temperatures (a double-loop path) before reaching the domain of the PG 1159 stars and eventually its terminal white dwarf cooling track. During these stages, the remnant is a quiescent heliumburning object that reaches the point of maximum $T_{\text {eff }}$ (the knee in the HR diagram) in about $15000 \mathrm{yr}$ after the occurrence of the last thermal pulse.

The evolution during the thermally pulsing phase is documented in Fig. 2. This figure shows the time dependence of the surface luminosity $\left(L_{\text {sur }}\right)$ and the hydrogen- and heliumburning luminosities ( $L_{\mathrm{CNO}}$ and $L_{\mathrm{He}}$, respectively) of our initially $2.7 M_{\odot}$ model star, where the time-scale is given in Myr from the main sequence (ZAMS). A total of 10 thermal pulses with an interpulse period of roughly $1.2 \times 10^{5} \mathrm{yr}$ have been computed before the remnant leaves the AGB as a result of the enhanced mass-loss. Note that the helium burning rate rises very steeply at the peak of each pulse, even during the first

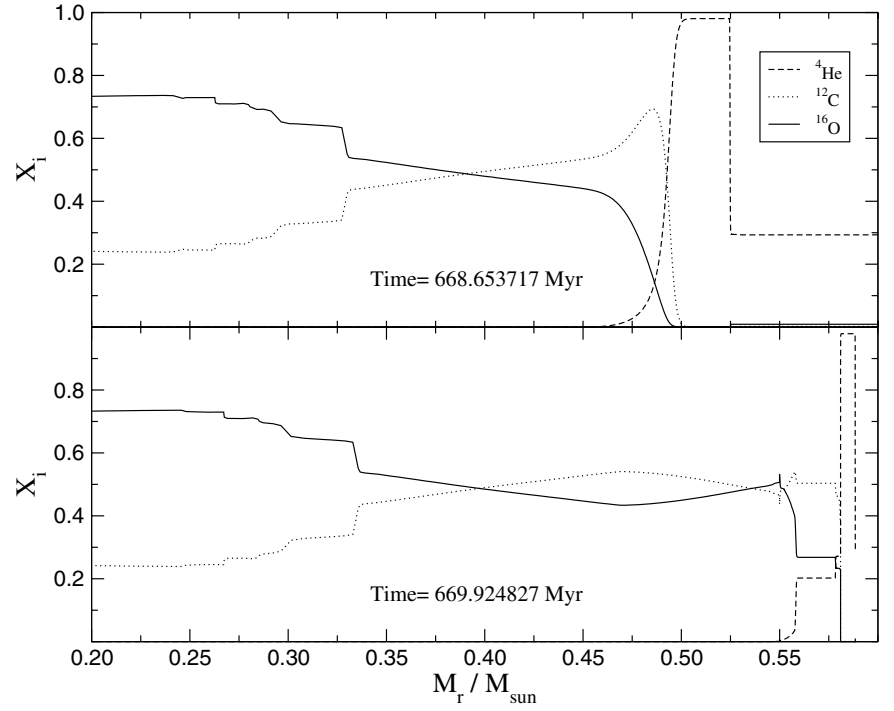

Fig. 3. Internal ${ }^{4} \mathrm{He},{ }^{12} \mathrm{C}$ and ${ }^{16} \mathrm{O}$ profiles for the $2.7 M_{\odot} \mathrm{DB}$ white dwarf progenitor shortly before the occurrence of the first thermal pulse on the AGB (upper panel) and at the onset of the last thermal pulse at high $T_{\text {eff }}$ (bottom panel). Overshoot episodes leave recognizable features both in the inner carbon-oxygen profile and in the intershell region that are particularly rich in oxygen. See text for details.

pulses. In our simulation, departure from the AGB takes place at such an advanced stage in the helium shell flash cycle that the post-AGB remnant will experience a last helium thermal pulse at high $T_{\text {eff }}$ values - see Fig. 1 and also the next section. This situation corresponds to the last thermal pulse and it is illustrated separately on the right panel of Fig. 2. During the last pulse, hydrogen-burning luminosity, due mainly to proton captures by ${ }^{12} \mathrm{C}$, reaches about $10^{8.5} L_{\odot}$.

The inner carbon, oxygen and helium distribution in the progenitor star as a function of the mass coordinate is shown in Fig. 3. The upper panel of the figure depicts the chemical profile at the onset of the thermally pulsing AGB phase, while the bottom panel illustrates the situation for the beginning of the last thermal pulse at high $T_{\text {eff }}$. The carbon and oxygen abundance distribution within the core is typical of situations in which extra mixing episodes beyond the fully convective core during central helium burning are allowed. Such extra mixing episodes, particularly core overshooting and/or semiconvection, have a large influence on the carbon and oxygen distribution in the core of white dwarfs (Straniero et al. 2003). In our simulation, the central oxygen mass abundance amounts to 0.724 when the helium convective core disappears. The shape of the carbon-oxygen profile, particularly the sharp variation around $M_{r} \approx 0.33 M_{\odot}$ induced by overshoot, is in agreement with that reported in Straniero et al. (2003). The importance of such extra mixing episodes, particularly core overshoot, for the pulsational properties of massive ZZ Ceti stars has recently been emphasized by Althaus et al. (2003) and Córsico et al. (2004). Note that during the thermally pulsing phase, the mass of the carbon-oxygen core increases considerably because the helium burning shell moves outwards. The signatures left by overshoot in the chemical profile during this stage is apparent. In particular, the overshoot region stretching below the 
short-lived helium-flash convection zone at pulse peak causes the intershell region below the almost pure helium buffer to be substantially enriched in oxygen. Another feature predicted by our calculations is the occurrence of the third dredge-up during the thermally pulsing phase. We will not discuss this aspect here - see Herwig (2000) for details. Suffice it to say that as a result of envelope overshoot, stretching down to the underlying carbon-rich layers, large amounts of carbon are dredged up to the surface after each thermal pulse. Indeed, the carbon-oxygen ratio increases from $\approx 0.25$ when entering the thermally pulsing phase to $\approx 0.34$ by the time the remnant departs from the AGB. We have checked the formation of a carbon star in our simulation by suppressing mass loss. We find in that case that a carbon-oxygen ratio larger than 1 occurs after the occurrence of the 15 th pulse.

Another prediction of our calculations is the formation of ${ }^{13} \mathrm{C}$ - and ${ }^{14} \mathrm{~N}$ - pockets at the base of the helium buffer after the end of the dredge-up phase. Indeed, during the third dredgeup, diffusive overshoot has formed a small region in which hydrogen from the envelope and carbon (resulting from helium burning) from the intershell region coexist in appreciable abundances. When this region heats up enough, hydrogen reignites giving rise to the formation of a ${ }^{14} \mathrm{~N}$-pocket with ${ }^{14} \mathrm{~N}$ abundances by mass of about 0.45 (the most abundant element in the pocket) and an underlying ${ }^{13} \mathrm{C}$-pocket with a maximum ${ }^{13} \mathrm{C}$ abundance of 0.06 . The mass range over which the ${ }^{14} \mathrm{~N}$ rich region extends amounts only to $\approx 2 \times 10^{-6}, M_{\odot}$. During the interpulse period, the ${ }^{13} \mathrm{C}$-pocket is radiatively burnt at relatively low temperatures (about $80 \times 10^{6} \mathrm{~K}$ ) before the onset of the next pulse via the reaction ${ }^{13} \mathrm{C}(\alpha, n){ }^{16} \mathrm{O}$, the main neutron source reaction in AGB stars. The ${ }^{14} \mathrm{~N}$-pocket is engulfed by the helium-flash convection zone during the next thermal pulse and burnt via the ${ }^{14} \mathrm{~N}(\alpha, \gamma){ }^{18} \mathrm{~F}\left(\beta^{+}, v\right){ }^{18} \mathrm{O}(\alpha, \gamma){ }^{22} \mathrm{Ne}$ chain that converts all the abundantly present ${ }^{14} \mathrm{~N}$ to ${ }^{22} \mathrm{Ne}$. In agreement with Herwig (2000), our calculations show that diffusive overshooting at the base of the convective envelope is a process that naturally leads to the formation of ${ }^{13} \mathrm{C}$ - and ${ }^{14} \mathrm{~N}$ - pockets, which is a fundamental issue regarding the formation of heavy elements through the slow neutron capture process - see, for instance, Lugaro et al. (2003) for a recent discussion.

\subsection{Evolution during the last thermal pulse and born again phase: The burning of the hydrogen envelope}

As a result of the mass-loss episodes, the mass of the hydrogen envelope is reduced to such an extent that the thermally pulsing progenitor star leaves the AGB and evolves into the planetary nebula regime at large $T_{\text {eff }}$ values. During this phase of the evolution, helium-shell burning increases gradually and when hydrogen burning becomes virtually extinct at the beginning of the white dwarf cooling branch, the post-AGB remnant experiences the last helium-shell flash that gives rise to the shortlived born-again episode. The mass of hydrogen that it is left in the very outer layers at the start of the last thermal pulse amounts to $7 \times 10^{-5} M_{\odot}$. Very few detailed numerical simulations through this complicated regime exist in the literature. Evolutionary calculations that include hydrogen and helium burning combined with time-dependent mixing have been performed initially by Iben \& MacDonald (1995) and by Herwig et al. (1999) for the situation in which diffusive overshooting is considered. Also, Lawlor \& MacDonald (2003) recently presented a grid of stellar evolutionary calculations for the bornagain phenomenon aimed to explain the observational characteristics of born-again stars. In what follows, we report the main predictions of our calculations for the born-again phase, particularly emphasizing the changes in the abundances of the different chemical species that take place during this brief evolutionary phase.

The Hertzsprung-Russell diagram focusing onto the last helium thermal pulse and the subsequent born-again evolutionary phase is displayed in Fig. 4. Selected evolutionary stages are labelled by letters along the evolutionary track. The inset shows the time-dependence of hydrogen- (CNO-cycle reactions) and helium-burning luminosities; again selected evolutionary stages are correspondingly labeled in this curve. Note that remarkable changes in the structure of the star take place on extremely short time-scales. For instance, it takes $0.4 \mathrm{yr}$ for the star to develop hydrogen-burning luminosities as high as $10^{8} L_{\odot}$ (point $\mathrm{C}$ in Fig. 4) after protons begin to be engulfed by the outward-growing helium-flash convection zone (point B). Most of the hydrogen envelope burning occurs between points $\mathrm{C}$ and $\mathrm{E}$ in about 1 month. Specifically, at point $\mathrm{E}$, after $0.9 \mathrm{yr}$ have elapsed from the onset of the last helium thermal pulse (point A), the mass of the residual hydrogen envelope has reduced to $10^{-7} M_{\odot}$. Between points $\mathrm{E}$ and $\mathrm{F}$, hydrogen burning becomes gradually extinct and the mass of hydrogen remaining in the star amounts to $1.3 \times 10^{-8} M_{\odot}$. Approximately 0.6 yr later, the remnant reaches the point of maximum effective temperature at $\log T_{\text {eff }}=4.8$ for the first time after the helium flash. Afterwards, the evolution proceeds into the red giant domain somewhat more slowly. In fact, the effective temperature decreases to $10000 \mathrm{~K}$ over a period of about $20 \mathrm{yr}$, and to $5200 \mathrm{~K}$ in about $50 \mathrm{yr}$ and the radius of the star increases to 30.2 and $125 R_{\odot}$, respectively.

Our born-again time-scale of $20-40 \mathrm{yr}$ is larger than the evolutionary time-scale of the born-again Sakurai object (V4334 Sgr), which has evolved from the pre-white dwarf stage into a AGB giant star in only about 6 yr. The evolutionary calculations of Herwig (2001), which are based on the standard MLT, also predict too large born-again time-scales (typically $350 \mathrm{yr}$ ). Herwig has found that the very short born-again evolutionary time of V4334 Sgr can be reproduced by stellar models if the convective mixing efficiency in the helium-flash convection zone is reduced by a factor of 100 below that obtained from the MLT. Although our evolution time-scales are longer than observed ones, they are much shorter than those obtained by Herwig (2001), but without invoking an additional reduction in the mixing efficiency. We remind the reader that in our calculation the double-diffusive MLT for fluids with composition gradients (Grossman \& Taam 1996) has been used ${ }^{3}$.

\footnotetext{
3 It is clear that the large difference in the evolutionary time scales between the both set of calculations cannot be entirely attributed to the fact that the mass value of our model is not strictly the same as that analyzed by Herwig (2001).
} 


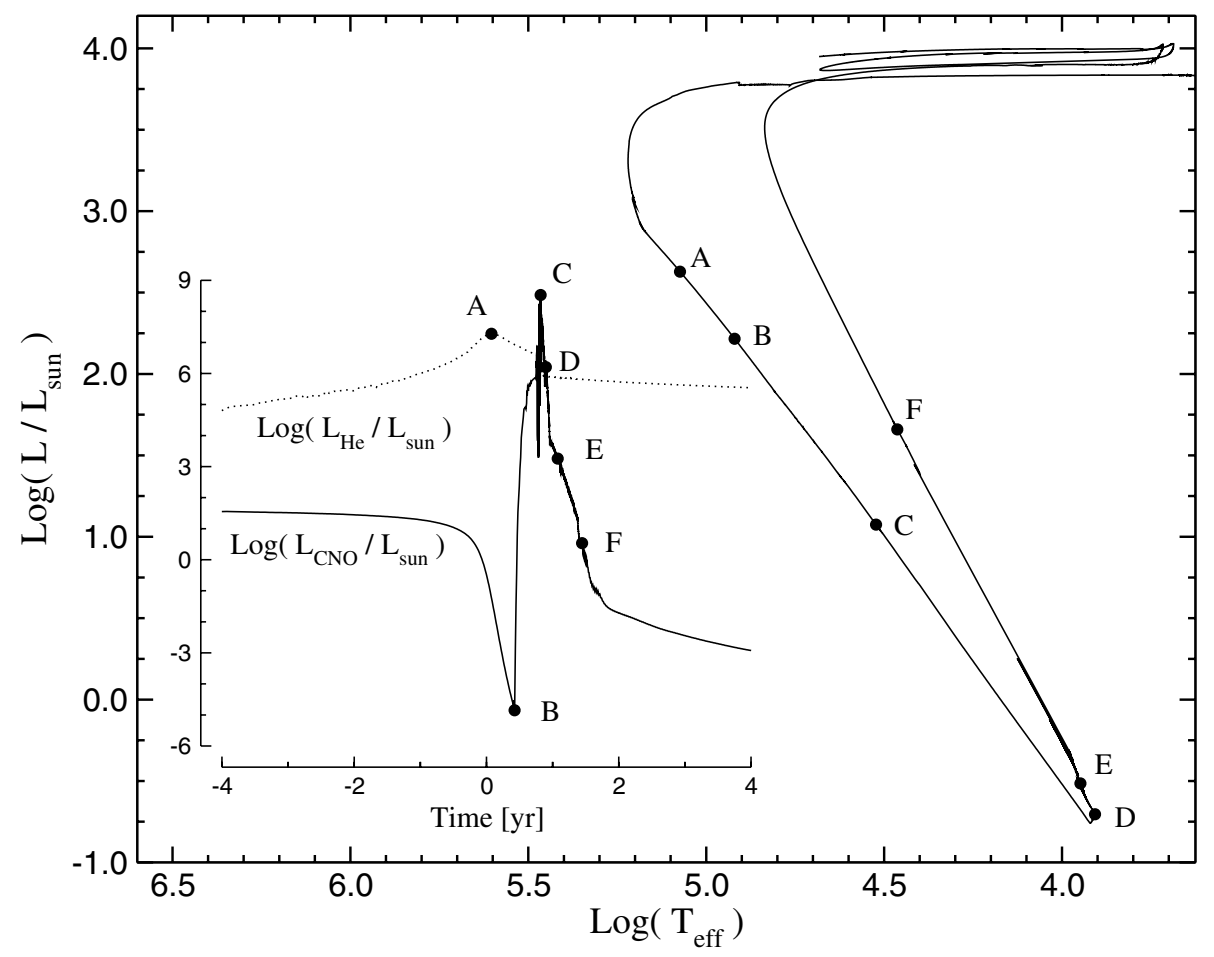

Fig. 4. Hertzsprung-Russell diagram for the evolutionary stages following the onset of the last helium thermal pulse that takes place at high effective temperatures (born-again episode) for our post-AGB remnant of $0.5885 M_{\odot}$. Note that the remnant experiences a second excursion (double loop) to the red giant region before evolving into its final white dwarf state. The left inset displays the time-dependence of hydrogenand helium-burning luminosities (solid and dotted lines, respectively) expressed in solar units. Selected stages are labeled by letters along the curves. In particular, points A and C correspond to the moment of maximum helium- and hydrogen-burning luminosities, respectively. At point $\mathrm{B}$, protons begin to be engulfed and burnt by the outward-growing, helium-flash convection zone. Between points $\mathrm{E}$ and $\mathrm{F}$, hydrogen burning becomes almost extinguished. The hydrogen content remaining in the star amounts to $1.3 \times 10^{-8} M_{\odot}$. Note the extremely short evolutionary time-scales characterizing this phase.

Another prediction of our calculations is the occurrence of a double-loop in the Hertzsprung-Russell diagram. That is, the star reaches red giant dimensions for a second time after the onset of the last helium thermal pulse and before finally returning to the white dwarf cooling track, a behaviour reported by Lawlor \& MacDonald (2003) and Herwig (2003) in the case of low convective mixing efficiency. In particular, this second return to the AGB takes about $350 \mathrm{yr}$ in our calculations. In the light of these results, we judge that a more comprehensive comparison between the standard mixing length theory and the double-diffusive mixing length theory deserves to be done. We postpone this to a forthcoming work.

During the last helium thermal pulse, profound changes in the chemical structure take place as a result of the vigorous nuclear processing and mixing episodes. Such changes are crucial for the subsequent evolution of the star. Because of the very short evolutionary time-scales characterizing this phase, a numerical treatment that consistently couples the equations of nuclear changes with the equations for time-dependent mixing processes, like the one adopted here, is indeed required for a realistic description of the abundance changes. A complete coverage of the inner chemistry variations that take place during this short evolutionary stage is provided in Fig. 5, which shows the chemical abundance distribution at four selected evolutionary stages during the last thermal pulse. Specifically, the abundances by mass of ${ }^{1} \mathrm{H},{ }^{4} \mathrm{He},{ }^{12} \mathrm{C},{ }^{13} \mathrm{C},{ }^{14} \mathrm{~N}$ and ${ }^{16} \mathrm{O}$ are plotted in terms of the outer mass fraction $q$. Grey and shaded regions mark the domains of convection and overshooting. Panel a shows the chemical stratification at the start of helium thermal pulse (point A in Fig. 4). In the outermost layers the chemical composition corresponds to that fixed by dredgeup episodes during the AGB phase. In the pure helium buffer, the relatively large abundance of ${ }^{14} \mathrm{~N}$ reflects the efficiency of hydrogen burning during previous evolutionary phases in processing CNO elements into ${ }^{14} \mathrm{~N}$. Because of the large amount of energy resulting from helium burning, an outward-growing convective region develops. In about $1 \mathrm{yr}$, the outer edge of this convective region reaches the base of the hydrogen-rich envelope. As a result, protons begin to be transported downwards into hotter and carbon-rich layers, where they are captured via the ${ }^{12} \mathrm{C}(p, \gamma){ }^{13} \mathrm{~N}$ reaction. The ensuing vigorous hydrogen burning forces the development of an entropy barrier. As a result, the original convective region is split at $\log q \approx-1.6$ in two distinct convective shells. The intermediate region is radiative, but also presents a salt-finger instability at its top. This situation is illustrated by panel b. Hydrogen and helium burning take place at the base of such convective layers. Note the large amount of ${ }^{13} \mathrm{C}$ in the convection zone powered by hydrogen burning (upper convection zone). In the hotter, heliumflash convection zone ${ }^{13} \mathrm{C}$ (and ${ }^{14} \mathrm{~N}$ ) is destroyed by $\alpha$ captures 


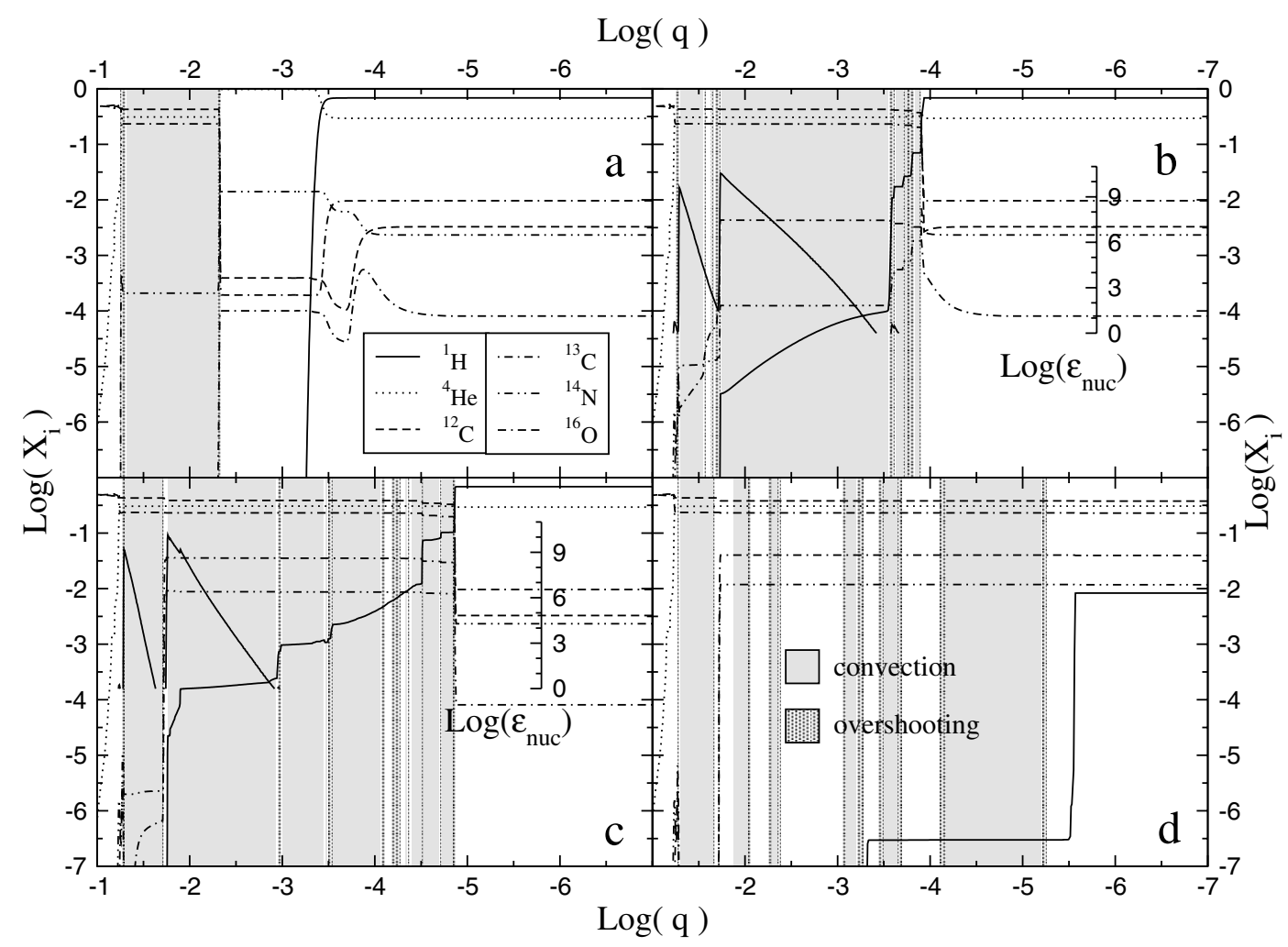

Fig. 5. Internal abundance distribution of ${ }^{1} \mathrm{H},{ }^{4} \mathrm{He},{ }^{12} \mathrm{C},{ }^{13} \mathrm{C},{ }^{14} \mathrm{~N}$ and ${ }^{16} \mathrm{O}$ as a function of the outer mass fraction $q$ for the $0.5885 M_{\odot}$ remnant at various selected epochs during the course of the last helium thermal pulse. Panel a) corresponds to the moment just before the occurrence of the peak helium-burning luminosity (point A in Fig. 4). The situation when the outward-moving, helium-flash convection zone has reached the base of the hydrogen-rich envelope (between points B and C in Fig. 4) is illustrated by panel b). Note that protons are ingested into deeper and hotter layers. As a result of the ongoing hydrogen burning a small radiative and salt-finger zone establishes at $\log q \approx-1.6$, which splits the convection zone into two. The situation two weeks later is visualized in panel c). During this time, the convection zone powered by hydrogen burning propagates outwards. Finally, panel d) illustrates the abundance profiles near point F in Fig. 4. Only traces of hydrogen remain after hydrogen burning. Nuclear energy release due to hydrogen and helium burning are also shown in panel b) and c).

and neutrons are released. Note as well that, as previously emphasized, the use of a simultaneous treatment of mixing and burning is indeed required during these stages, as reflected by the hydrogen profile.

Panel c depicts the situation two weeks later. During this time, the outer edge of the upper convection zone propagates further outwards in mass, mixing downwards protons from the original (unprocessed) hydrogen-rich envelope. Note both the persistence of the small salt-finger region separating the two convection zones and the increase in the ${ }^{13} \mathrm{C}$ and ${ }^{14} \mathrm{~N}$ abundances in the upper convection zone due to hydrogen processing. The surface and upper convection zones merge temporarily (point E in Fig. 4), causing the convectively unstable region to extend from the hydrogen-burning zone essentially to the surface of the star. Only a very thin unprocessed hydrogen-rich envelope remains, which is expected to be diluted by surface convection when the star returns to the AGB. Finally, panel d shows the abundance profiles near point $F$ in Fig. 4. Here, hydrogen burning is virtually extinct and the remaining hydrogen mass amounts to only $1.3 \times 10^{-8} M_{\odot}$. Note also that traces of hydrogen are present in layers even as deep as $3 \times 10^{-4} M_{\odot}$ below the stellar surface. Interestingly enough, the content of ${ }^{13} \mathrm{C}$ left in the whole star is sizeable and amounts to $4.5 \times 10^{-4} M_{\odot}$, that is $4-5$ orders of magnitude larger than the hydrogen mass of the star.

\subsection{Evolution towards the PG 1159 state}

After the last thermal pulse, as the star evolves back to giant dimensions, either convective dilution or mass loss during the quiescent helium-burning phase (Iben et al. 1983) are expected to erode the tiny layer of original envelope material, exposing the underlying hydrogen-deficient layers. Thus, after the second loop in the HR diagram, the remnant evolves to the region of the PG 1159 stars with a surface chemical composition similar to that shown in panel $d$ of Fig. 5. Specifically, in the outer layers, ${ }^{4} \mathrm{He},{ }^{12} \mathrm{C}$ and ${ }^{16} \mathrm{O}$ are by far the dominant species with abundance by mass $\left({ }^{4} \mathrm{He},{ }^{12} \mathrm{C},{ }^{16} \mathrm{O}\right)=(0.306,0.376,0.228)$. Amongst the main remaining constituents are ${ }^{13} \mathrm{C},{ }^{14} \mathrm{~N}$ and ${ }^{22} \mathrm{Ne}$ with mass fractions of $4,1.2$ and $2.1 \%$, respectively. ${ }^{13} \mathrm{C}$ and ${ }^{14} \mathrm{~N}$ are present from the outermost layers down to the base of the former upper convection zone at $0.01 M_{\odot}$ below the stellar surface. At deeper layers, these chemical species have been already depleted via $\alpha$ captures. The large mass fraction of oxygen is an indication of the occurrence of diffusive overshooting during the thermally pulsing AGB phase (see also Herwig et al. 1999). The final surface composition of our 


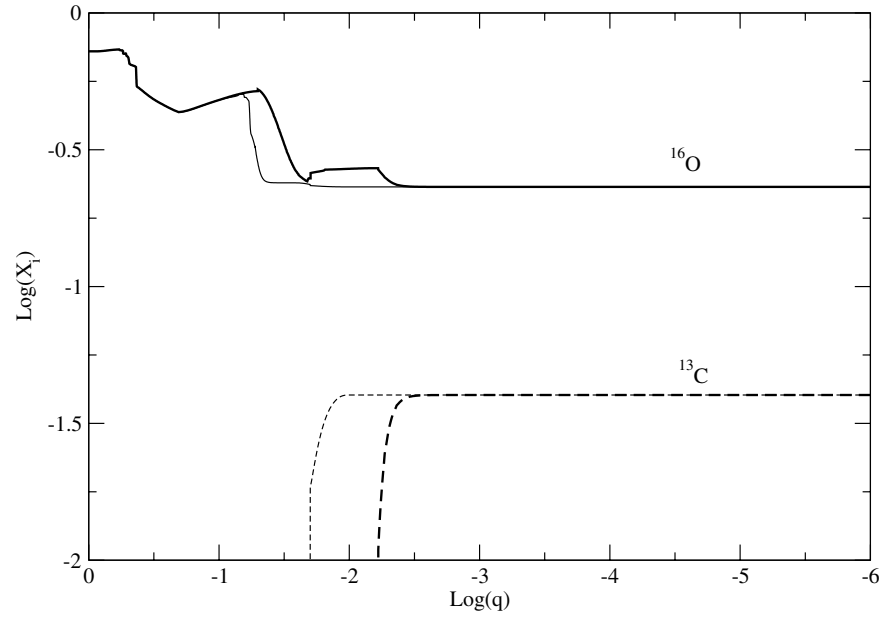

Fig. 6. Evolution of the internal abundances of ${ }^{13} \mathrm{C}$ and ${ }^{16} \mathrm{O}$ (dashed and solid lines respectively) across the PG 1159 domain in terms of the outer mass fraction $q$ for the $0.5885 M_{\odot}$ remnant. Thin and thick lines correspond, respectively, to a model at $T_{\text {eff }}=71000 \mathrm{~K}$ before the point of maximun effective temperature in the HR diagram and $T_{\text {eff }}=125000 \mathrm{~K}$ below that point. Note the formation of a bump in the oxygen distribution as a result of $\alpha$ captures by ${ }^{13} \mathrm{C}$.

models is also in line with surface abundance patterns observed in most hot, hydrogen-deficient post AGB stars such as PG 1159 stars and central stars of planetary nebulae of spectral type WC (Koesterke \& Hamann 1997; Dreizler \& Heber 1998; Werner 2001). The remarkable agreement between the ${ }^{14} \mathrm{~N}$ abundance predicted by our calculations and that detected by Dreizler \& Heber (1998) in five out of nine PG 1159 stars strongly supports the hypothesis that these stars would be AGB descendants that have experienced a born-again episode. As we have already seen, mixing and burning of protons in the heliumflash convective region are indeed required to synthesize ${ }^{14} \mathrm{~N}$ in a carbon- and oxygen-rich environment.

As the evolution proceeds through the domain of the PG 1159 stars, changes in the chemical composition take place as a result of nuclear burning. Figure 6 is an example of this. In a region stretching from $3 \times 10^{-3}$ to $10^{-2} M_{\odot}$ below the stellar surface, a bump in the oxygen distribution is built up as a result of ${ }^{13} \mathrm{C}$ burning via the ${ }^{13} \mathrm{C}(\alpha, n){ }^{16} \mathrm{O}$ reaction. This is a consequence of the fact that before the hydrogen-deficient remnant reaches the point of maximum effective temperature in the HR diagram, the temperature at the tail of the ${ }^{13} \mathrm{C}$ distribution exceeds $85 \times 10^{6} \mathrm{~K}$, which is high enough for the ${ }^{13} \mathrm{C}(\alpha, n){ }^{16} \mathrm{O}$ reaction to operate. During the early PG 1159 stage, the helium content in the star is reduced from $8.5 \times 10^{-3}$ to $5.2 \times 10^{-3} M_{\odot}$ as a result of helium burning. This value is the amount of helium with which the star enters the white dwarf domain after helium burning becomes extinct. Because we have not invoked any additional mass loss after the born-again episode, the amount of helium left in the white dwarf $\left(5.2 \times 10^{-3} M_{\odot}\right)$ should be considered as an upper limit for the particular case analyzed in this work (see next section for the effect of mass-loss episodes during the hot stages of post-AGB evolution).

In Fig. 7, we show as a function of time the luminosity contributions due to helium burning $\left(L_{\mathrm{He}}\right)$, neutrino losses $\left(L_{\mathrm{neu}}\right)$, surface luminosity $\left(L_{\mathrm{sur}}\right)$ and gravothermal energy release $\left(L_{\mathrm{g}}\right)$ for the hydrogen-deficient $0.5885 M_{\odot}$ remnant. The abscissa covers the time span from the pre-white dwarf state, before the maximum $T_{\text {eff }}$ point in the HR diagram, to beyond the domain of the variable DB white dwarfs. Luminosities are given in solar units; the age is counted from the moment at which the remnant reached $T_{\text {eff }}=70000 \mathrm{~K}$. At early times it is mostly helium burning that contributes to the surface luminosity of the star. This is true for the first 15000-20000 yr of evolution, shortly after the remnant reaches the maximum $T_{\text {eff }}$ value, and begins to decrease as the star approaches the white dwarf domain. Afterwards, the contribution of helium burning declines steeply and the evolution of the star is dictated essentially by neutrino losses and the release of gravothermal energy. At the $T_{\text {eff }}$ value characterizing PG 1159-035 ( $\log T_{\text {eff }} \simeq 5.12$ ), gravothermal energy is the main energy source of the star. But, for the coolest pulsators in the GW Vir strip, neutrino losses exceed photon luminosity. This is an important feature since it allows to use cool pulsating PG 1159 stars to constrain neutrino emission processes from measurements of the rate of period change see O'Brien et al. (1998) and O'Brian \& Kawaler (2000) for a discussion of this issue. Note that neutrino losses constitute the primary cooling mechanism over a period of about $1.5 \times 10^{7} \mathrm{yr}$, during which the star evolves through the temperature range $90000 \mathrm{~K} \gtrsim T_{\text {eff }} \gtrsim 25000 \mathrm{~K}$. The lower temperature limit corresponds to the domain of the hot pulsating DB white dwarfs. Thus, these variable stars should also be potentially useful to place constraints on plasmon neutrino emissivity, as recently emphasized by Winget et al. (2004).

In Fig. 8 we show the surface gravity-effective temperature diagram for our post born-again $0.5885 M_{\odot}$ sequence together with observational data for hydrogen-deficient PG 1159 and WC stars, as taken from Werner et al. (1997) and Werner (2001). In particular, the pulsating nitrogen-rich PG 1159 stars analyzed by Dreizler \& Heber (1998) - namely, PG 1159-035, PG 2131+066, PG 1707+427 and PG 0122+200 - are consistent with a stellar mass somewhat lower than that characterizing our post born-again sequence.

\subsection{White dwarf evolution}

Once helium burning becomes virtually extinct at $T_{\text {eff }} \approx$ $100000 \mathrm{~K}$, after $100000 \mathrm{yr}$ of evolution from the last helium thermal pulse, the hydrogen-deficient remnant settles on its terminal cooling track. Here, its chemical abundance distribution will be strongly modified by the various diffusion processes acting during white dwarf evolution. This can be seen in Fig. 9 which illustrates the mass abundances of ${ }^{4} \mathrm{He},{ }^{12} \mathrm{C},{ }^{16} \mathrm{O},{ }^{13} \mathrm{C}$, ${ }^{14} \mathrm{~N}$ and ${ }^{22} \mathrm{Ne}$ for the $0.5885 M_{\odot}$ white dwarf as a function of the outer mass fraction at various epochs characterized by values of $\log L / L_{\odot}$ and $\log T_{\text {eff }}$ (the corresponding values are given in parentheses for each of the panels). Panel a shows the chemical stratification at the start of the cooling track after the star has reached the point of maximum $T_{\text {eff }}$ at high luminosities. In the outermost layers the chemical composition corresponds essentially to that emerging from the mixing and burning events during the last helium thermal pulse and 


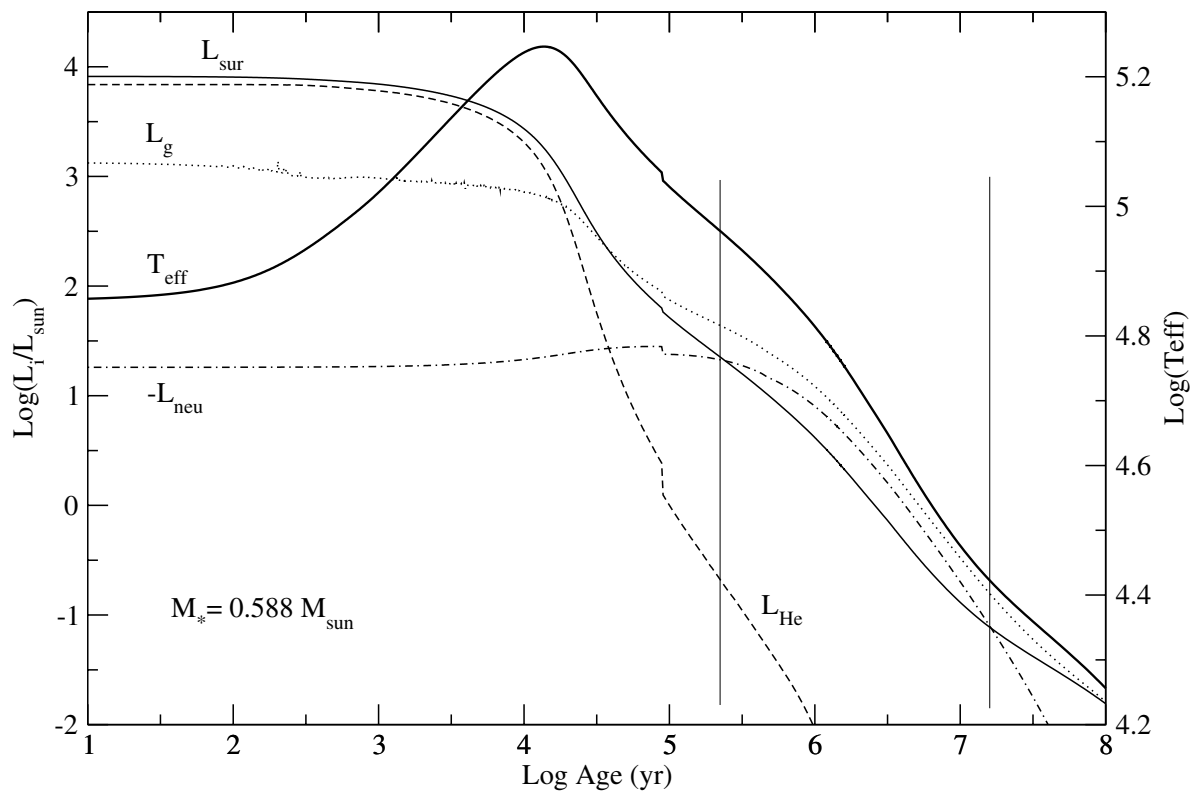

Fig. 7. Time-dependence of the different luminosity contributions (in solar units) for the post born again $0.5885 M_{\odot}$ remnant of the evolution of the $2.7 M_{\odot}$ star: surface luminosity, $L_{\text {sur }}$, helium burning luminosity, $L_{\mathrm{He}}$, neutrino losses, $L_{\text {neu }}$ and rate of gravothermal (compressional plus thermal) energy release $L_{\mathrm{g}}$. The evolution of the effective temperature (right scale) is also plotted. Time is in years counted from the moment at which the remnant reaches $T_{\text {eff }}=70000 \mathrm{~K}$. The vertical lines bracket the domain where neutrino losses exceed photon luminosity.

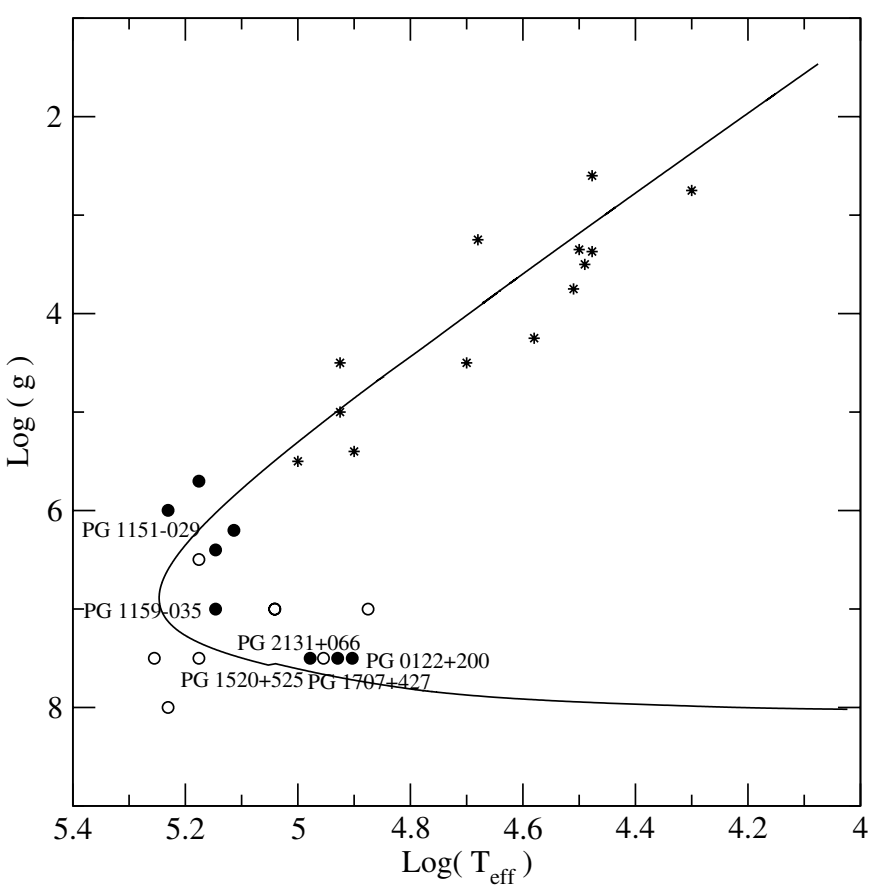

Fig. 8. Evolution of the surface gravity (in cgs units) as a function of the effective temperature for the post born-again $0.5885 M_{\odot}$ remnant and its comparison with some observed hot hydrogen-deficient stars. Filled (open) circles denote pulsating (non-pulsating) PG 1159 stars and asterisks observed WC stars (Werner et al. 1997; Werner 2001).

subsequent born-again episode. Rapidly, gravitational settling causes helium to float to the surface and heavier elements to sink. In fact, in about $1.5 \times 10^{6} \mathrm{yr}$ the star develops a pure helium envelope of nearly $6 \times 10^{-8} M_{\odot}$ (panel b). By the time the domain of the variable DBs is reached (after $1.77 \times 10^{7} \mathrm{yr}$ of white dwarf evolution; panel c) gravitationally-induced diffusion has led to the development of a double-layered chemical structure characterized by a pure helium envelope of $2.3 \times$ $10^{-6} M_{\odot}$ atop an intermediate remnant shell rich in helium, carbon and oxygen, the relics of the last helium thermal pulse (see previous section). About $3.7 \times 10^{8} \mathrm{yr}$ later, the white dwarf reaches the domain of the helium-rich, carbon-contaminated DQ white dwarfs. The corresponding chemical stratification is shown in panel d. Even at such an advanced stage, the star is characterized by a double-layered structure. In particular, the pure helium envelope amounts to $1.9 \times 10^{-5} M_{\odot}$. Note the significant carbon enrichment in the surface layers as a result of convective dredge-up of the carbon diffusive tail by the superficial helium convection zone (see later in this section). The neutron excess characterizing ${ }^{13} \mathrm{C}$ (and also ${ }^{22} \mathrm{Ne}$ ) partially explains the fact that this element appreciably diffuses downwards. It is clear from these figures that diffusion processes substantially alter the chemical abundance distribution in the course of white dwarf evolution.

The effect of element diffusion on the main chemical constituents can best be visualized in Fig. 10, particularly the formation of the double-layered chemical structure. The mass abundances of ${ }^{4} \mathrm{He},{ }^{12} \mathrm{C}$ and ${ }^{16} \mathrm{O}$ are shown as a function of the outer mass fraction for the $0.5885 M_{\odot}$ white dwarf remnant at three evolutionary stages. The chemical profiles shortly after the remnant reaches the point of maximum $T_{\text {eff }}$ in the HR diagram are represented with thin lines of various patterns. Later evolutionary stages around the domain of the DB instability strip $\left(\log T_{\text {eff }}=4.41\right.$ and 4.28$)$ are represented with normal and heavy lines. We stress again that the helium content that is left in the star at the start of the cooling branch, after helium burning has virtually ceased, amounts to $5.2 \times 10^{-3} M_{\odot}$. 

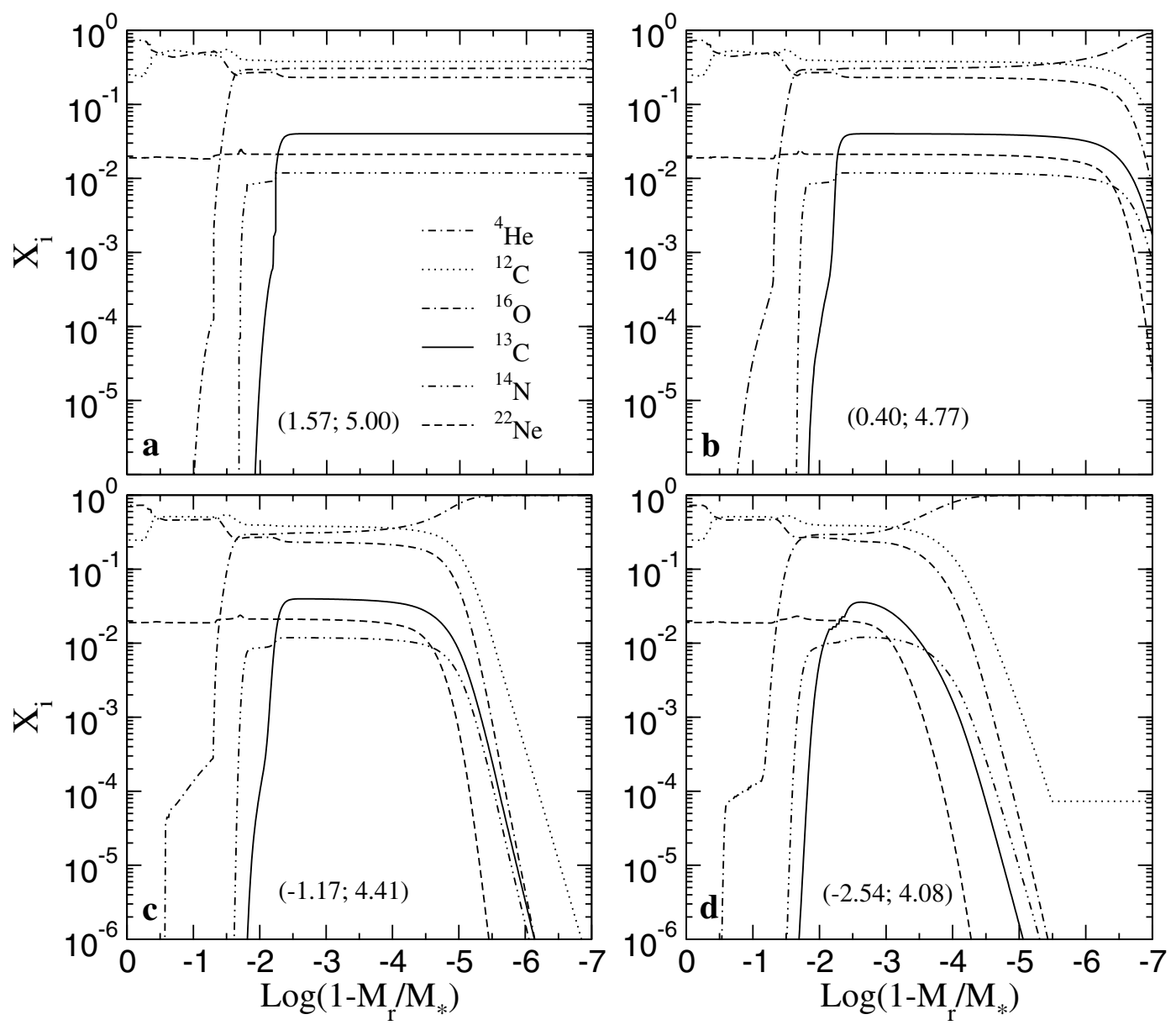

Fig. 9. Abundance by mass of ${ }^{4} \mathrm{He},{ }^{12} \mathrm{C},{ }^{16} \mathrm{O},{ }^{13} \mathrm{C},{ }^{14} \mathrm{~N}$ and ${ }^{22} \mathrm{Ne}$ in terms of the outer mass fraction for the $0.5885 M_{\odot}$ remnant at four selected white-dwarf evolutionary stages, characterized by values of $\log L / L_{\odot}$ and $\log T_{\text {eff }}$ (the corresponding values are given in parentheses). Panel a) corresponds to the start of the cooling branch, panel c) to an evolutionary stage during the DB instability strip and panel d) to the domain of the carbon-enriched DQ white dwarfs. Panel d) illustrates the significant carbon enrichment in the surface layers as a result of convective dredge-up of the carbon diffusive tail by the superficial helium convection zone. It is clear that element diffusion strongly modifies the internal chemical profiles of the hydrogen-deficient white dwarf.

Because we have not invoked any additional mass loss during the hot post-AGB stages or early during the cooling branch, the quoted value for the final helium mass should be considered as an upper limit for the particular case of evolution analyzed here. The diffusion-induced double-layered structure at the domain of the pulsating DBs is easily recognizable. Another feature worthy of comment is the mixing episode that takes place in the region below the intershell zone around $\log q=-1$. This region is characterized by a inward-decreasing mean molecular weight induced by the occurrence of overshooting during the AGB thermally pulsing phase. The resulting salt-finger mixing is responsible for the redistribution of the chemical species in that region, as is apparent from Fig. 10.

We have extended the scope of our evolutionary calculations down to the domain of the carbon-enriched DQ white dwarfs, thus covering the possible evolutionary connection PG 1159-DB-DQ. DQ white dwarfs have $T_{\text {eff }}$ values below $13000 \mathrm{~K}$ (Weidemann \& Koester 1995) and are characterized by the presence of trace amounts of carbon in their atmospheres with abundances by number relative to helium, $\log \left(n_{\mathrm{C}} / n_{\mathrm{He}}\right)$, ranging from -7.3 to -1.5 (MacDonald et al. 1998). The presence of traces of carbon observed in these stars is widely believed to result from convective dredge-up of the carbon diffusive tail by the superficial helium convection zone (Pelletier et al. 1986; Koester et al. 1982). By the time the DQ domain is reached, our white dwarf models have developed a double-layered chemical structure with a pure helium mantle of $1.9 \times 10^{-5} M_{\odot}$, which is almost an order of magnitude as massive as the helium mantle characterizing our models at the beginning of the DB instability strip. However, it is less massive than required for heavy elements to be abundantly dredged-up to the surface by the inwards-growing superficial convection zone. This fact is reflected in Fig. 11 which shows the surface abundance by number relative to ${ }^{4} \mathrm{He}$ of ${ }^{12} \mathrm{C},{ }^{16} \mathrm{O},{ }^{13} \mathrm{C}$ and ${ }^{14} \mathrm{~N}$ as a function of the effective temperature. As the white dwarf cools, the base of the convection zone moves deeper into the star, with the consequent further enrichment with heavy elements of the outer layers. Note that ${ }^{12} \mathrm{C}$ is by far the most abundant dredged-up element, with abundances far exceeding the low carbon abundances observed in many DQ (see next section). Note also that our calculations predict the presence of ${ }^{13} \mathrm{C}$ and ${ }^{14} \mathrm{~N}$, abundantly created during the last helium thermal 


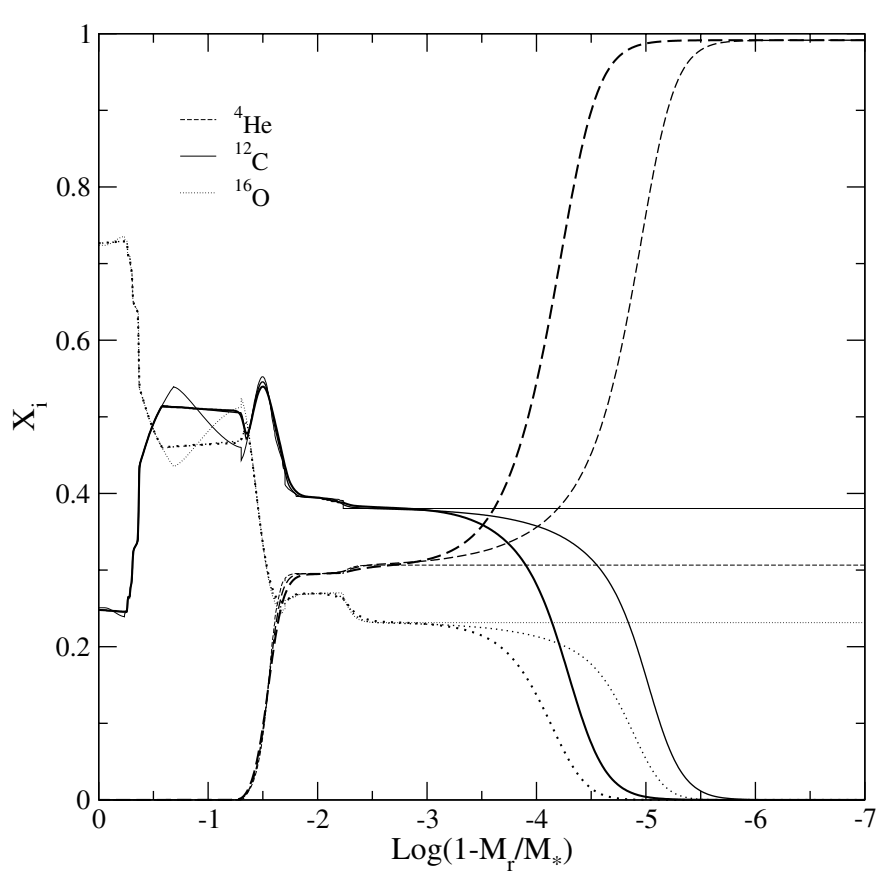

Fig. 10. Abundances by mass of ${ }^{4} \mathrm{He},{ }^{12} \mathrm{C}$ and ${ }^{16} \mathrm{O}$ as a function of the outer mass fraction for the $0.5885 M_{\odot}$ white-dwarf remnant having a final helium content of $5.2 \times 10^{-3} M_{\odot}$. Initial profiles at the start of the cooling branch $\left(\log T_{\mathrm{eff}}=5.0\right)$ are plotted with thin lines. Later stages, denoted with normal and heavy lines, correspond to $\log T_{\text {eff }}=4.41$ and 4.28 respectively.

pulse, as well as ${ }^{16} \mathrm{O}$ in the atmospheres of DQ white dwarfs. Finally, ${ }^{22} \mathrm{Ne}$ has diffused so deep into the star (see Fig. 9, panel d) that it is expected not to be dredged-up to the surface of these stars.

For the sake of completeness, we provide in Table 1 some relevant quantities for our post born-again $0.5885 M_{\odot}$ sequence. Specifically, we list from left to right the effective temperature, the photon luminosity (in solar units), the age (in years) counted from the moment at which the remnant reaches $T_{\text {eff }}=10000 \mathrm{~K}$ (after the occurrence of the double loop at high luminosities), the stellar radius (in $\mathrm{cm}$ ), the surface gravity and the helium-burning and neutrino luminosities (both in solar units). The tabulation covers the stages following the end of the born-again episode to the domain of the DQ white dwarfs at low $T_{\text {eff }}$ values.

\section{Discussion}

The calculations presented in this work cover the whole evolutionary stages involved in the formation of DB white dwarfs via the born-again scenario starting on the ZAMS. In particular, our calculations have followed the model star to very advanced stages of evolution down to the domain of the helium-rich carbon-contaminated DQ, the supposed cooler descendants of DBs. Hence, the study opens the possibility of assessing the evolutionary connection PG 1159-DB-DQ (Fontaine \& Brassard 2002) in the framework of complete evolutionary calculations that take into account a detailed treatment of the physical processes that lead to the formation of hydrogen-deficient white dwarfs. In this sense, the results presented here reinforce

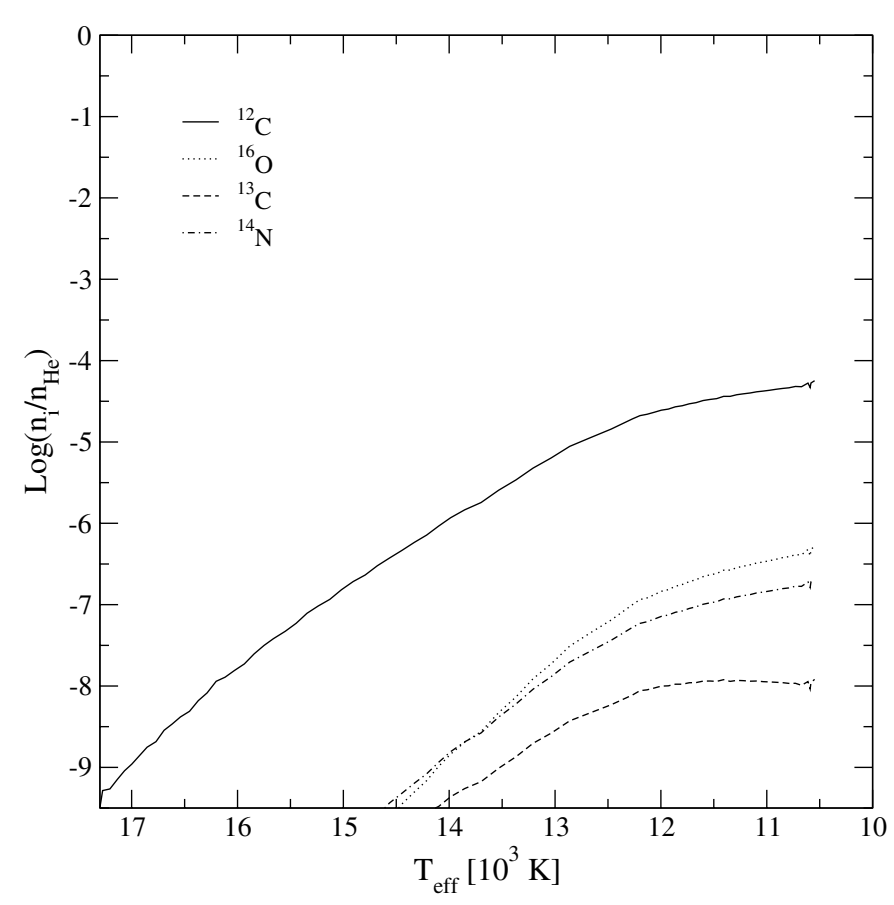

Fig. 11. Logarithm of the number density of surface ${ }^{12} \mathrm{C},{ }^{16} \mathrm{O},{ }^{13} \mathrm{C}$ and ${ }^{14} \mathrm{~N}$ relative to that of ${ }^{4} \mathrm{He}$ as a function of the effective temperature.

the conclusions arrived at in Fontaine \& Brassard (2002) and Althaus \& Córsico (2004) about the presence of a diffusivelyevolving double-layered chemical structure in pulsating DB white dwarfs. Our results show that element diffusion proceeds very efficiently in these stars, causing the thickness of the pure helium envelope to increase by almost an order of magnitude by the time the DQ domain is reached, as compared with the situation at the beginning of the DB instability strip.

A novel aspect of our work is the fact that the calculation of the evolutionary stages prior to the white-dwarf formation allows us to make sound predictions of the surface abundances expected in the DQ stars. In this connection, we find that ${ }^{12} \mathrm{C}$ is by far the most abundant element that is convectively dredgedup to the outer layers, but with abundances far exceeding the low carbon abundances detected in many DQs (MacDonald et al. 1998). If we push our results to their limits (see below), then the plausibility of an evolutionary link between PG 1159 and DQ stars with low detected carbon abundance appears to be unclear if convective dredge-up is responsible for the observed carbon in DQs. Our study also predicts the presence of trace amounts of ${ }^{16} \mathrm{O},{ }^{13} \mathrm{C}$ and ${ }^{14} \mathrm{~N}$ in the atmospheres of these white dwarfs.

However, it is conceivable that the occurrence of mass-loss episodes before and during the hot PG 1159 stage could alter some aspects of the above-mentioned results considerably. In this regard, recent observational evidence hints at the possibility that post-AGB mass-loss episodes could markedly reduce the mass of the helium-rich envelope. Specifically, the immediate PG 1159 predecessors, the [WC] stars, which range roughly between $T_{\mathrm{eff}}=30000$ and $140000 \mathrm{~K}$ in the highluminosity region of the early post-AGB evolution are known to have mass-loss rates of about $10^{-5.5}$ to $10^{-6.5} M_{\odot} / \mathrm{yr}$ that generally decrease with increasing $T_{\text {eff }}$. In addition, numerous 
Table 1. Selected stages for our post-born again $0.5885 M_{\odot}$ sequence.

\begin{tabular}{|c|c|c|c|c|c|c|}
\hline $\log T_{\text {eff }}[\mathrm{K}]$ & $\log \left(\frac{L}{L_{\odot}}\right)$ & $\log \tau[\mathrm{yr}]$ & $\log R_{*}[\mathrm{~cm}]$ & $\log g\left[\mathrm{~cm} \mathrm{~s}^{-2}\right]$ & $\log \left(\frac{L_{\mathrm{He}}}{L_{\odot}}\right)$ & $\log \left(-\frac{L_{\nu}}{L_{\odot}}\right)$ \\
\hline 4.0466 & 3.9940 & 0.3629 & 12.2700 & 1.3524 & 3.9908 & 1.2555 \\
\hline 4.1044 & 3.9928 & 0.7537 & 12.1539 & 1.5848 & 3.9884 & 1.2555 \\
\hline 4.1493 & 3.9916 & 0.9283 & 12.0634 & 1.7657 & 3.9865 & 1.2555 \\
\hline 4.2061 & 3.9898 & 1.0999 & 11.9489 & 1.9948 & 3.9836 & 1.2554 \\
\hline 4.2586 & 3.9878 & 1.2323 & 11.8429 & 2.2067 & 3.9804 & 1.2554 \\
\hline 4.3060 & 3.9856 & 1.3402 & 11.7470 & 2.3985 & 3.9770 & 1.2554 \\
\hline 4.3586 & 3.9826 & 1.4526 & 11.6402 & 2.6120 & 3.9724 & 1.2554 \\
\hline 4.4053 & 3.9795 & 1.5480 & 11.5453 & 2.8019 & 3.9675 & 1.2553 \\
\hline 4.4552 & 3.9756 & 1.6473 & 11.4435 & 3.0056 & 3.9611 & 1.2553 \\
\hline 4.5000 & 3.9714 & 1.7348 & 11.3519 & 3.1888 & 3.9542 & 1.2549 \\
\hline 4.5537 & 3.9655 & 1.8392 & 11.2416 & 3.4092 & 3.9442 & 1.2548 \\
\hline 4.6017 & 3.9593 & 1.9345 & 11.1425 & 3.6075 & 3.9328 & 1.2548 \\
\hline 4.6500 & 3.9519 & 2.0355 & 11.0421 & 3.8083 & 3.9200 & 1.2547 \\
\hline 4.7014 & 3.9424 & 2.1563 & 10.9346 & 4.0233 & 3.9003 & 1.2548 \\
\hline 4.7515 & 3.9315 & 2.3064 & 10.8290 & 4.2345 & 3.8734 & 1.2549 \\
\hline 4.8010 & 3.9221 & 2.5381 & 10.7251 & 4.4422 & 3.8384 & 1.2559 \\
\hline 4.8508 & 3.9156 & 2.7410 & 10.6225 & 4.6476 & 3.8360 & 1.2585 \\
\hline 4.9003 & 3.9007 & 2.8811 & 10.5159 & 4.8607 & 3.8366 & 1.2603 \\
\hline 4.9500 & 3.8804 & 2.9975 & 10.4064 & 5.0797 & 3.8240 & 1.2621 \\
\hline 5.0002 & 3.8549 & 3.1382 & 10.2933 & 5.3060 & 3.7937 & 1.2649 \\
\hline 5.0503 & 3.8224 & 3.2890 & 10.1768 & 5.5390 & 3.7610 & 1.2693 \\
\hline 5.1005 & 3.7774 & 3.4503 & 10.0538 & 5.7848 & 3.7121 & 1.2758 \\
\hline 5.1502 & 3.7134 & 3.6242 & 9.9224 & 6.0478 & 3.6410 & 1.2868 \\
\hline 5.2001 & 3.6081 & 3.8226 & 9.7700 & 6.3525 & 3.5196 & 1.3038 \\
\hline 5.2466 & 3.2645 & 4.1517 & 9.5053 & 6.8819 & 3.0875 & 1.3515 \\
\hline 5.2001 & 2.6917 & 4.4184 & 9.3118 & 7.2688 & 2.1510 & 1.4052 \\
\hline 5.1507 & 2.3614 & 4.5701 & 9.2454 & 7.4018 & 1.5060 & 1.4294 \\
\hline 5.1001 & 2.0635 & 4.7480 & 9.1978 & 7.4970 & 0.9067 & 1.4455 \\
\hline 5.0525 & 1.8007 & 4.9492 & 9.1615 & 7.5694 & 0.3861 & 1.4480 \\
\hline 5.0018 & 1.5620 & 5.1430 & 9.1435 & 7.6055 & -0.2817 & 1.3667 \\
\hline 4.9512 & 1.2973 & 5.4055 & 9.1125 & 7.6675 & -0.7823 & 1.3114 \\
\hline 4.9011 & 1.0430 & 5.6464 & 9.0855 & 7.7215 & -1.2399 & 1.1936 \\
\hline 4.8517 & 0.7988 & 5.8578 & 9.0621 & 7.7684 & -1.6699 & 1.0408 \\
\hline 4.8003 & 0.5493 & 6.0509 & 9.0403 & 7.8120 & -2.2074 & 0.8467 \\
\hline 4.7502 & 0.3148 & 6.2117 & 9.0232 & 7.8461 & -2.9096 & 0.6406 \\
\hline 4.7011 & 0.0919 & 6.3536 & 9.0100 & 7.8726 & -3.7778 & 0.4345 \\
\hline 4.6511 & -0.1312 & 6.4955 & 8.9984 & 7.8957 & -4.8588 & 0.2115 \\
\hline 4.6015 & -0.3477 & 6.6278 & 8.9893 & 7.9139 & -6.0306 & -0.0137 \\
\hline 4.5540 & -0.5528 & 6.7585 & 8.9817 & 7.9291 & -7.3201 & -0.2475 \\
\hline 4.5045 & -0.7646 & 6.9069 & 8.9748 & 7.9429 & -8.9760 & -0.5207 \\
\hline 4.4558 & -0.9713 & 7.0723 & 8.9689 & 7.9547 & -99.0000 & -0.8388 \\
\hline 4.4035 & -1.1917 & 7.2858 & 8.9633 & 7.9660 & -99.0000 & -1.2737 \\
\hline 4.3540 & -1.3994 & 7.5261 & 8.9584 & 7.9757 & -99.0000 & -1.8102 \\
\hline 4.3030 & -1.6132 & 7.7839 & 8.9536 & 7.9853 & -99.0000 & -2.4829 \\
\hline 4.2505 & -1.8326 & 8.0244 & 8.9489 & 7.9948 & -99.0000 & -3.2861 \\
\hline 4.2024 & -2.0322 & 8.2128 & 8.9454 & 8.0018 & -99.0000 & -4.1214 \\
\hline 4.1527 & -2.2370 & 8.3788 & 8.9423 & 8.0080 & -99.0000 & -4.9905 \\
\hline 4.1027 & -2.4419 & 8.5257 & 8.9398 & 8.0128 & -99.0000 & -5.8260 \\
\hline 4.0500 & -2.6564 & 8.6674 & 8.9380 & 8.0166 & -99.0000 & -7.9835 \\
\hline 4.0232 & -2.7651 & 8.7349 & 8.9373 & 8.0180 & -99.0000 & -99.0000 \\
\hline
\end{tabular}

hot, low-gravity PG 1159 stars, have been reported (Koesterke \& Werner 1998) to exhibit mass-loss rates ranging from $10^{-7}$ to $10^{-8} M_{\odot} /$ yr. Finally, tentative evidence for the persistence of mass-loss rates of the same order along the hot end of the white dwarf cooling sequence and down to the domain of hot helium-rich white dwarfs has also been presented (Werner 2001). Notably, the existence of PG 1159 stars with a helium content as low as $1 \times 10^{-3} M_{\odot}$ has been suggested by 
asteroseismology in at least one of these stars with a stellar mass of $0.6 M_{\odot}$ (Kawaler \& Bradley 1994), thus implying the occurrence of mass-loss during the evolution towards the PG 1159 phase. In principle, these mass-loss rates are large enough to leave their signatures in the further evolution of these stars.

To assess the possible implications of such mass-loss events for white dwarf evolution, we have extended our calculations by considering two further evolutionary sequences in which mass loss is addressed during the hot post-AGB evolutionary stages. Specifically, we invoke extreme constant mass-loss rates of $5 \times 10^{-8}$ and $1 \times 10^{-7} M_{\odot} / \mathrm{yr}$ along the hot post-AGB track from the low-gravity domain at $T_{\text {eff }} \approx 115000 \mathrm{~K}$ sustained all the way down to the hot white dwarf cooling sequence at about $T_{\text {eff }}=80000 \mathrm{~K}$. As a result, we find that the helium content that eventually survives in the star amounts to $1.4 \times 10^{-3}$ and $9.4 \times 10^{-4} M_{\odot}$, respectively. The total mass lost by the star amounts to about $0.013 M_{\odot}$ in both cases. Most of the mass is lost after the star has reached the point of maximum $T_{\text {eff }}$. We note that mass loss uncovers deep regions in the star where the chemical composition varies with depth. Thus, the outer layer chemical stratification after the end of mass loss at the start of the white dwarf cooling track looks somewhat different from the situation in which mass loss is not considered. In particular, the external chemical interfaces are markedly smoother. The implications for the chemical profiles expected during the DB instability strip are clearly visualized with the help of Figs. 12 and 13 for the mass-loss rates of $5 \times 10^{-8}$ and $10^{-7} M_{\odot} / \mathrm{yr}$, respectively. Note in particular that, in the case of the lowest helium content, a single and not a double-layered profile is more appropriate to describe the outer layer chemical structure of pulsating DB white dwarfs. This is in line with the conclusion arrived at in Althaus \& Córsico (2004) that if post born-again DB white dwarf progenitors are formed with a helium content smaller than $10^{-3} M_{\odot}$ a doublelayered structure is not expected by the time the star reaches the red edge of the DB instability strip. Thus, the calculations presented here place that conclusion on a more solid basis. It is also clear that the initial chemical profile is different according to whether mass loss actually occurs or not, an aspect which is expected to affect the pulsational properties of variables DBs.

Even in the case of extreme mass-loss rates of $10^{-7} M_{\odot} / y r$, the helium envelope is not completely removed. However, the rate is high enough to erode any vestige of ${ }^{14} \mathrm{~N}$ before the remnant reaches $T_{\text {eff }} \approx 88000 \mathrm{~K}$. The detection of abundant ${ }^{14} \mathrm{~N}$ in some coolest PG 1159 stars (for instance in PG 0122+200 at $T_{\text {eff }} \approx 80000 \mathrm{~K}$; see Dreizler \& Heber 1998) makes the persistence of such extreme mass loss rather unlikely.

Additionally, we investigate the consequences of mass-loss episodes during the hot post-AGB phase for the surface abundances expected in DQ stars. The predicted surface ${ }^{12} \mathrm{C}$ abundance together with the observed carbon abundance in DQ atmospheres are shown in Fig. 14. Our results indicate that if DQ white dwarfs are the descendants of the post-born again PG 1159 stars, then their surface carbon abundance is not expected to exhibit a marked dependence on the helium content with which the white dwarf is formed. In particular, note that the carbon abundance far exceeds the low carbon abundances

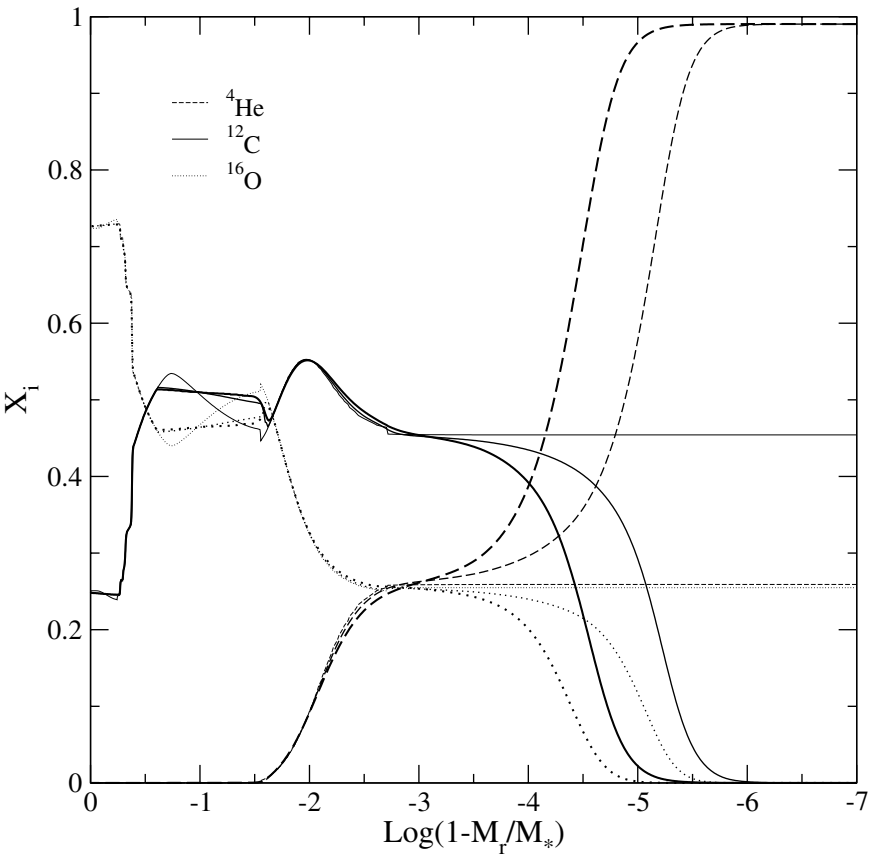

Fig. 12. Same as Fig. 10 but for a final helium content of $1.4 \times 10^{-3} M_{\odot}$. The initial profiles at the start of the cooling track $\left(\log T_{\text {eff }}=4.89\right)$ are plotted with thin lines. Later stages, denoted with normal and heavy lines, correspond to $\log T_{\text {eff }}=4.407$ and 4.29 respectively.

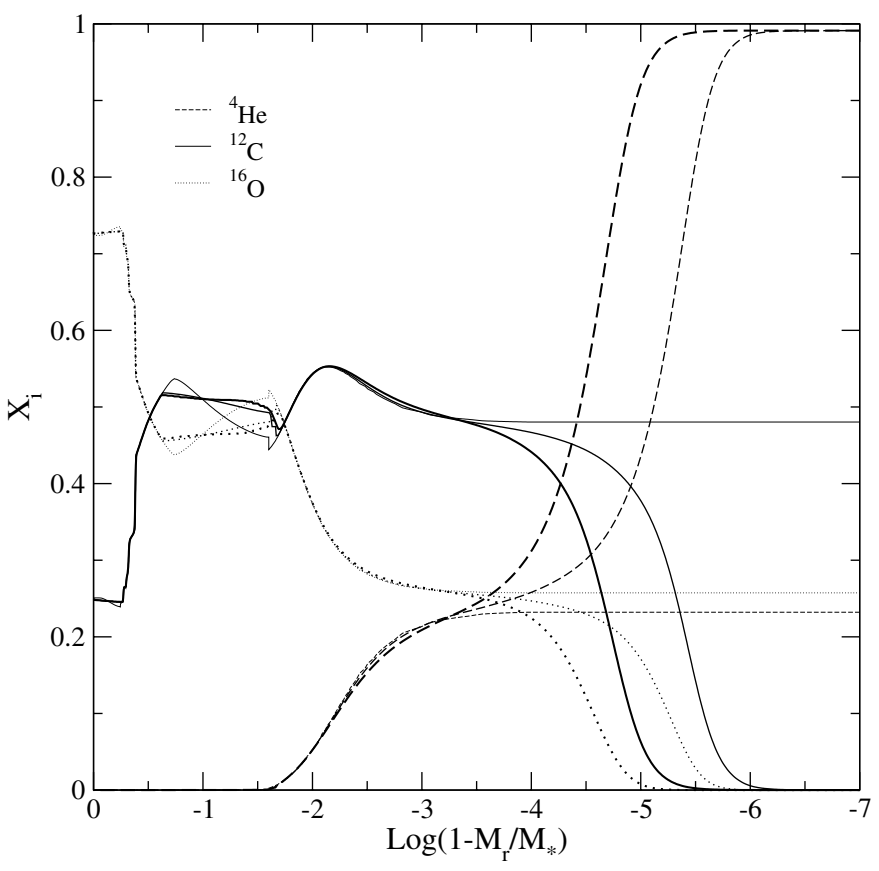

Fig. 13. Same as Fig. 10 but for a final helium content of $9.4 \times 10^{-4} M_{\odot}$. The initial profiles at the start of the cooling branch $\left(\log T_{\text {eff }}=4.93\right)$ are plotted with thin lines. Later stages, denoted with normal and heavy lines, correspond to $\log T_{\mathrm{eff}}=4.42$ and 4.31 respectively.

detected in numerous DQs. This prompts us to suggest that the DQs with low carbon abundance cannot be linked to the PG 1159 stars if canonical convective dredge-up is the source of carbon for such DQs. Instead, they appear more likely to be good candidates for an evolutionary connection that link them 


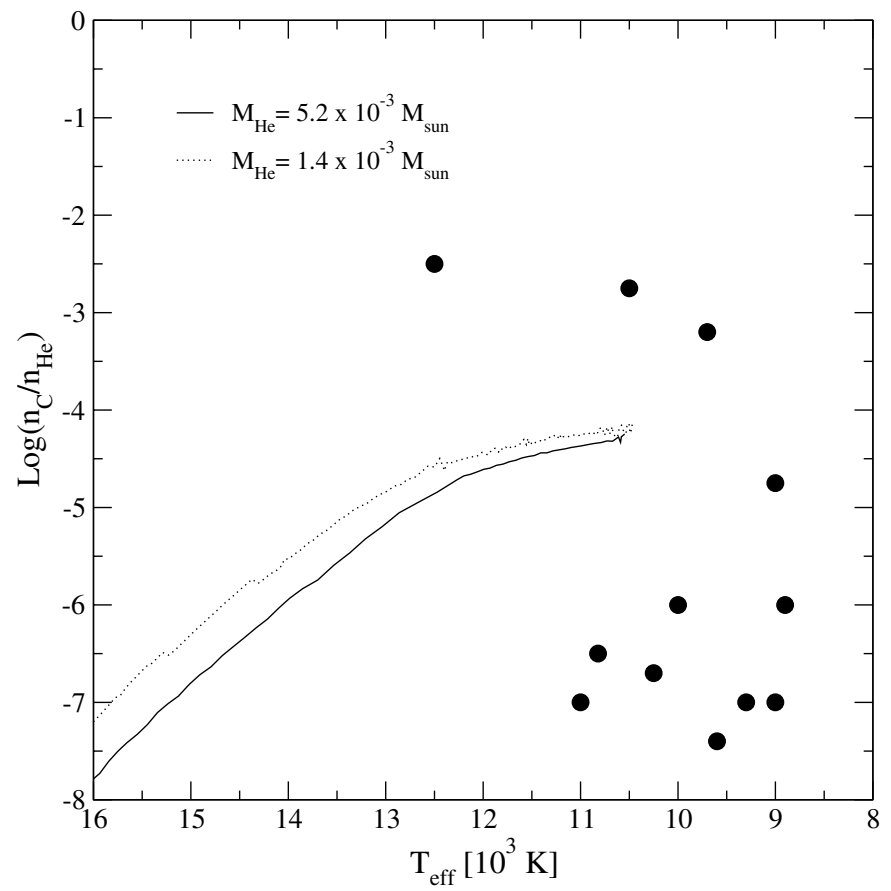

Fig. 14. Number density of surface ${ }^{12} \mathrm{C}$, relative to that of ${ }^{4} \mathrm{He}$ as a function of the effective temperature. Solid and dotted lines correspond to the white dwarf remnant with helium content of $5.2 \times 10^{-3}$ and $1.4 \times 10^{-3} M_{\odot}$, respectively. Filled circles refer to observed carbon abundance as taken from MacDonald et al. (1998).

with stars that have somehow avoided the AGB thermally pulsing phase such as the hydrogen-poor $\mathrm{AGB}$ manqué or the $\mathrm{RCrB}$ stars, with normal carbon abundances.

High mass-loss rates like those suggested by observation have consequences for the evolutionary time-scales of the star. We find that for $\dot{M}=5 \times 10^{-8}$ and $10^{-7} M_{\odot} / \mathrm{yr}$, the star takes about $2.25 \times 10^{5}$ and $1.5 \times 10^{5} \mathrm{yr}$, respectively, to evolve from $T_{\text {eff }}=117000 \mathrm{~K}$ down to $T_{\text {eff }}=81000 \mathrm{~K}$. This is markedly shorter than the time $\left(4 \times 10^{5} \mathrm{yr}\right)$ needed for the star to evolve across the same $T_{\text {eff }}$ interval in the absence of mass loss. This reduction in the evolutionary time-scales induced by mass-loss is expected to yield high rates of period change in pulsating GW Vir stars, thus helping to partially overcome the discrepancy between theory and the observed value in the pulsating star PG 1159-035 (Costa et al. 1999).

In closing this section, we comment on the role played by the small amount of hydrogen that survives proton burning during the last helium thermal pulse. As previously mentioned, the hydrogen content eventually remaining in the star amounts to $1.3 \times 10^{-8} M_{\odot}$, with traces of this element reaching layers as deep as $3 \times 10^{-4} M_{\odot}$ below the stellar surface. After the bornagain phase, the total time spent by the remnant in the red-giant domain amounts to about 300-400 yr. Consequently, a massloss rate larger than $10^{-6} M_{\odot} / \mathrm{yr}$ would be enough to remove, during this time interval, the last vestiges of hydrogen-rich material left in the star. The development of a surface lacking any hydrogen could also occur during further PG 1159 evolution as a result of hydrogen burning and the persistence of a constant wind of about $10^{-9} M_{\odot} / \mathrm{yr}$ down to the hot white dwarf cooling branch. In the results presented thus far, we have assumed

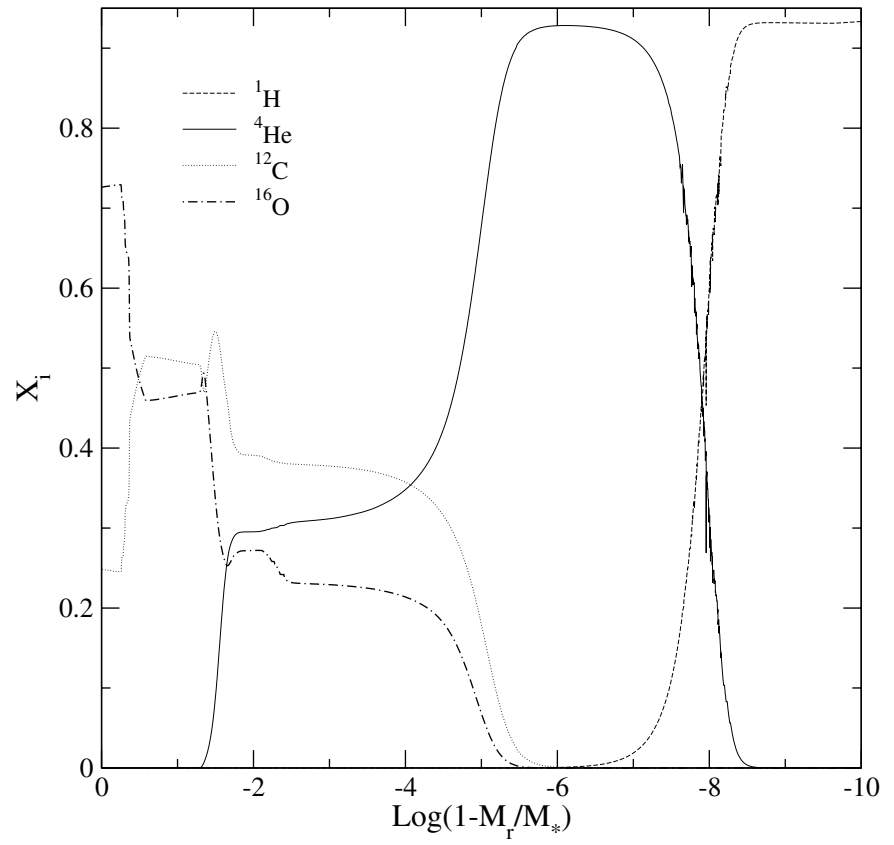

Fig. 15. Abundance by mass of ${ }^{1} \mathrm{H},{ }^{4} \mathrm{He},{ }^{12} \mathrm{C}$ and ${ }^{16} \mathrm{O}$ as a function of the outer mass fraction for the $0.5885 M_{\odot}$ white dwarf remnant at $T_{\text {eff }}=27000 \mathrm{~K}$. Here, post-born again mass-loss episodes have been neglected. Thus, traces of hydrogen remain in the star at the beginning of white dwarf evolution. Note that element diffusion leads to the formation of a DA white dwarf with a pure hydrogen envelope mass of about $5 \times 10^{-9} M_{\odot}$. In this particular simulation, we have neglected the diffusion of minor species such as ${ }^{13} \mathrm{C}$ and ${ }^{14} \mathrm{~N}$.

that it is indeed the actual course of events. From the opposite point of view, however, that is, in the case of much weaker or less persistent mass-loss events, we would expect the formation of a DA white dwarf with a very thin hydrogen envelope as a result of element diffusion. This is indeed borne out by Fig. 15, which illustrates the chemical abundance distribution by the time the white dwarf reaches $T_{\text {eff }}=27000 \mathrm{~K}$. The inner hydrogen has diffused outwards and has formed a pure hydrogen envelope of mass $5 \times 10^{-9} M_{\odot}$, if the extreme situation of no mass loss is assumed, turning the white dwarf into one of the DA type. Thus, under these circunstances, it is conceivable that the born-again episode could also give rise to DA white dwarfs with very thin hydrogen envelopes.

\section{Conclusions}

In this paper we have studied some relevant aspects of the evolution of hydrogen-deficient white dwarfs (hereafter referred to as DB white dwarfs) by means of new evolutionary models based on a complete and self-consistent treatment of the evolutionary stages prior to white dwarf formation. Specifically, we focused on DB white dwarfs, the progenitors of which have experienced a born-again episode, that is, a very late helium thermal pulse on the early white-dwarf cooling branch after hydrogen burning has almost ceased. The inclusion of a timedependent scheme for the simultaneous treatment of nuclear evolution and mixing processes due to convection, salt fingers and diffusive overshoot has allowed us to perform a detailed 
study of the abundance changes throughout all of the evolutionary phases, particularly during the thermally pulsing AGB state and the extremely short-lived phase of the born-again episode, for which the assumption of instantaneous mixing becomes completely inadequate. Our calculations made use the doublediffusive mixing length theory of convection for fluids with composition gradients (Grossman \& Taam 1996). The study can thus be considered as an assessment of its performance in low-mass stars. In particular, we have concentrated on the evolution of an initially $2.7 M_{\odot}$ star from the zero-age main sequence through the thermally pulsing and mass-loss phases to the white dwarf stage.

As for the core helium burning and thermally pulsing AGB phases, our main results are:

- The inner carbon-oxygen profile exhibits a sharp variation around $M_{\mathrm{r}} \approx 0.33 M_{\odot}$ induced by overshoot.

- Our models experience the third dredge-up during the AGB. Also, overshooting from below the short-lived helium-flash convection region at pulse peak causes the intershell region below the helium-rich buffer to be enriched with abundant oxygen, in agreement with Herwig (2000).

- We find that pockets of ${ }^{13} \mathrm{C}$ and ${ }^{14} \mathrm{~N}$ are formed at the base of the helium buffer after the end of the dredge-up phase. During the interpulse period, the ${ }^{13} \mathrm{C}$-pocket is radiatively burnt, whilst the ${ }^{14} \mathrm{~N}$-pocket is engulfed by the helium-flash convection zone during the next thermal pulse.

For the born-again evolution:

- The convection theory of Grossman \& Taam (1996) appears to provide markedly shorter born-again time-scales than previous computations; this without invoking any reduction in the convective efficiency. However, they are still longer than the evolutionary time-scale of the born-again Sakurai object. This is a preliminary result, however, which should be explored in more detail.

- After the born-again phase, we find the occurrence of a double-loop path in the HR diagram; that is, the star reaches giant dimensions for the second time after the onset of the last helium thermal pulse and before finally returning to the white dwarf cooling track. The amount of hydrogen remaining in the star is $1.3 \times 10^{-8} M_{\odot}$. Most of the hydrogen envelope burning occurs in about 1 month.

- After the born-again episode, ${ }^{4} \mathrm{He},{ }^{12} \mathrm{C}$ and ${ }^{16} \mathrm{O}$ are by far the dominant surface species with mass abundances of $\left({ }^{4} \mathrm{He},{ }^{12} \mathrm{C},{ }^{16} \mathrm{O}\right)=(0.306,0.376,0.228)$. Amongst the main remaining constituents ${ }^{13} \mathrm{C},{ }^{14} \mathrm{~N}$ and ${ }^{22} \mathrm{Ne}$ are found with mass fractions of $4,1.2$ and $2.1 \%$, respectively. This is in agreement with surface abundance patterns observed in most hydrogen-deficient post-AGB stars such as PG 1159 stars. The high ${ }^{14} \mathrm{~N}$ surface abundance we find is also in line with that detected by Dreizler \& Heber (1998) in some PG 1159 stars.

- During the early PG 1159 stage, about $3 \times 10^{-4} M_{\odot}$ of ${ }^{13} \mathrm{C}$ is processed via the ${ }^{13} \mathrm{C}(\alpha, n){ }^{16} \mathrm{O}$ reaction. Also, the helium content in the star is reduced from $8.5 \times 10^{-3}$ to $5.2 \times 10^{-3} M_{\odot}$ as a result of helium burning.
Additionally, the inclusion of the various processes responsible for element diffusion expected during the white dwarf regime has enabled us to extend the scope of our calculations to the domain of the helium-rich, carbon contaminated DQ white dwarfs, and more importantly, to assess the plausibility of the evolutionary connection PG 1159-DB-DQ. In this regard, our main conclusions are:

- Diffusion processes lead to the formation of a doublelayered chemical structure during the DB evolution. In particular, by the time the domain of the pulsating DBs is reached, above the helium-, carbon- and oxygen- rich intershell, there exists a pure helium layer of $2.3 \times 10^{-6} M_{\odot}$, which increases to $1.9 \times 10^{-5} M_{\odot}$ when the star reaches the DQ domain.

- As a result of convective dredge-up at low $T_{\text {eff }}$, we find superficial ${ }^{12} \mathrm{C}$ with abundances far exceeding the low carbon abundances observed in many DQs. Our results suggest that the DQs with observed low carbon abundances cannot be linked to the PG 1159 stars if canonical convective dredge-up is the source of carbon for such DQs.

In closing, we believe that our study could be relevant for pulsational applications since it provides a self-consistent description of the composition profile for both the core and the envelope. As recently shown by Metcalfe et al. (2003) this is an important aspect as far as asteroseismological inferences about pulsating DB white dwarfs are concerned. Additionally, our new models constitute a physically sound frame for answering some open questions about pulsating GW Vir stars, the hotter pulsating predecesors of variable DB stars (Gautschy 1997). Our full evolutionary models could help to shed new light on the physical processes responsible for the driving of pulsations in these stars. Work in this direction is in progress.

Detailed tabulations of our post-born again model are freely available at the following URL:

http://www. fcaglp.unlp.edu.ar/evolgroup/

Acknowledgements. We warmly acknowledge T. Blöcker and K. Werner for sending us some reprints central to this work. We also acknowledge A. Gautschy for a careful reading of the manuscript. We thank our referee, whose suggestions and comments improve the original version of this paper. L.G.A also acknowledges the Spanish MCYT for a Ramón y Cajal Fellowship. A.M.S. has been supported by the W. M. Keck Foundation through a grant to the IAS and by the National Science Foundation through the grant PHY-0070928. Part of this work has been supported by the Instituto de Astrofísica La Plata, by the MCYT grant AYA2002-4094-C03-01, by the CIRIT and by the European Union FEDER funds.

\section{References}

Alexander, D. R., \& Ferguson, J. W. 1994, ApJ, 437, 879

Althaus, L. G., \& Córsico, A. H. 2004, A\&A, 417, 1115

Althaus, L. G., Serenelli, A. M., Córsico, A. H., \& Montgomery, M. H. 2003, A\&A, 404, 593

Anders, E., \& Grevesse, N. 1986, Geochim. Csomochim. Acta, 53, 197

Angulo, C., Arnould, M., Rayet, M., et al. 1999, Nucl. Phys. A, 656, 3

Arnett, W. D., \& Truran, J. W. 1969, ApJ, 157, 339 
Blöcker, T. 1995, A\&A, 297, 727

Blöcker, T. 2001, Ap\&SS, 275, 1

Burgers, J. M. 1969, Flow Equations for Composite Gases, Academic, New York

Caughlan, G. R., \& Fowler, W. A. 1988, Atom. Data Nucl. Data Tables, 40, 290

Córsico, A. H., Althaus, L. G., Montgomery, M. H., García-Berro, E., \& Isern, J. 2005, A\&A, 429, 277

Costa, J. E. S., Kepler, S. O., \& Winget, D. E. 1999, ApJ, 522, 973

Dehner, B. T., \& Kawaler, S. D. 1995, ApJ, 445, L141

Dreizler, S., \& Heber, U. 1998, A\&A, 334, 618

Dreizler, S., \& Werner, K. 1996, A\&A, 314, 217

Fontaine, G., \& Brassard, P. 2002, ApJ, 581, L33

Fujimoto, M. Y. 1977, PASJ, 29, 331

Gautschy, A. 1997, A\&A, 320, 811

Gautschy, A., \& Althaus, L. G. 2002, A\&A, 382, 141

Grossman, S. A., \& Taam, R. E. 1996, MNRAS, 283, 1165

Grossman, S. A., Narayan, R., \& Arnett, D. 1993, ApJ, 407, 284

Herwig, F. 2000, A\&A, 360, 952

Herwig, F. 2001, ApJ, 554, L71

Herwig, F. 2003, in Planetary Nebulae: their evolution and role in the Universe, ed. S. Kwok, M. Dopita and R. Sutherland, IAU Symp. 209, ASP Conf. Ser., 111

Herwig, F., Blöcker, T., Schönberner, D., \& El Eid, M. 1997, A\&A, 324, L81

Herwig, F., Blöcker, T., Langer, N., \& Driebe, T. 1999, A\&A, 349, L5

Iben, I. Jr., \& MacDonald, J. 1995, in White Dwarfs, Proc. of the 9th European Workshop on white dwarfs, ed. D. Koester, \& K. Werner (Berlin: Springer), 443, 48

Iben, I. Jr., Kaler, J. B., Truran, J. W., \& Renzini, A. 1983, ApJ, 264, 605

Iglesias, C. A., \& Rogers, F. J. 1996, ApJ, 464, 943

Itoh, N. 1997, in Advances in Stellar Evolution, Proc. of the Workshop Stellar Ecology, ed. R. T. Rood \& A. Renzini (Cambridge, UK: Cambridge Univ. Press), 185
Itoh, N., Hayashi, H., \& Kohyama, Y. 1994, ApJ, 436, 418

Kawaler, S. D., \& Bradley, P. A. 1994, ApJ, 427, 415

Koester, D., Weidemann, V., \& Zeidler-K.T., E. M. 1982, A\&A, 116, 147

Koesterke, L., \& Hamann, W. R. 1997, A\&A, 320, 91

Koesterke, L., \& Werner, K. 1998, ApJ, 500, L55

Lawlor, T. M., \& MacDonald, J. 2003, ApJ, 583, 913

Lugaro, M., Herwig, F., Lattanzio, J. C., Gallino, R., Straniero, O. 2003, ApJ, 586, 1305

MacDonald, J., Hernanz, M., \& José, J. 1998, MNRAS, 296, 523

Magni, G., \& Mazzitelli, I. 1979, A\&A, 72, 134

Mazzitelli, I., D’Antona, F., \& Ventura, P. 1999, A\&A, 348, 846

Metcalfe, T. S., Montgomery, M. H., \& Kawaler, S. D. 2003, MNRAS, 344, L88

O’Brien, M. S., \& Kawaler, S. D. 2000, ApJ, 539, 372

O’Brien, M. S., Vauclair, G., Kawaler, S. D., et al. 1998, ApJ, 495, 458

Pelletier, C., Fontaine, G., Wesemael, F., Michaud, G., \& Wegner, G. 1986, ApJ, 307, 242

Schönberner, D. 1979, A\&A, 79, 108

Schönberner, D. 1996, in Hydrogen-Deficient Stars, ed. C. S. Jeffery, \& U. Heber, ASP Conf. Ser., 96, 433

Schwarzschild, M., \& Härm, R. 1965, ApJ, 142, 855

Straniero, O., Domínguez, I., Imbriani, G., \& Piersanti, L. 2003, ApJ, 583,878

Unglaub, K., \& Bues, I. 2000, A\&A, 359, 1042

Ventura, P., D’Antona, F., \& Mazzitelli, I. 1999, ApJ, 524, L111

Weidemann, V., \& Koester, D. 1995, A\&A, 297, 216

Werner, K. 2001, Ap\&SS, 275, 27

Werner, K., Dreizler, S., Heber, U., et al. 1997, Rev. Mod. Astron., 10, 219

Winget, D. E., Sullivan, D. J., Metcalfe, T. S., Kawaler, S. D., \& Montgomery, M. H. 2004, ApJ, 602, L109 